
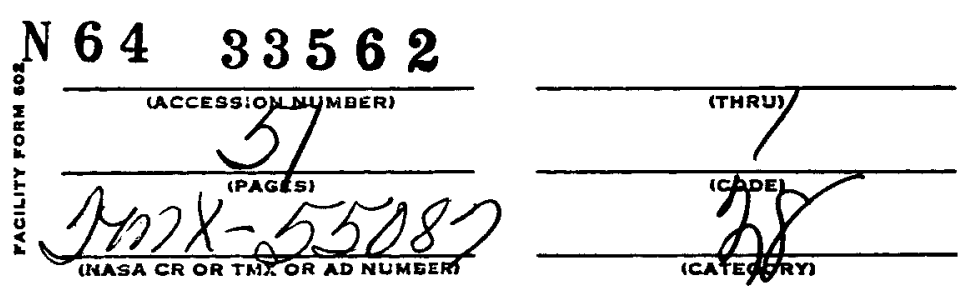

\title{
STUDIES OF SOLAR PROTONS WITH EXPLORERS XII AND XIV
}

\author{
BY \\ D. A. BRYANT \\ T. L. CLINE \\ U. D. DESAI \\ AND \\ F. B. MCDONALD \\ JULY 1964
}

$\frac{n}{0}$

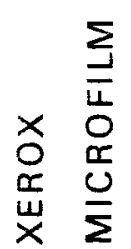

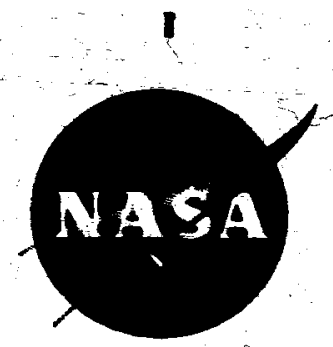

\section{GODDARD SPACE FLIGHT CENTER GREENBELT, MARYLAND'}

(Goddard Energetic Particles Preprint Series) 


\title{
STUDIES OF SOLAR PROTONS WITH EXPLORERS XII AND XIV \\ D. A. BRYANT*, T. L. CLINE, U. D. DESAI** AND F. B. McDONALD \\ Goddard Space Flight Center, Greenbelt, Maryland
}

\begin{abstract}
33562
\end{abstract}

Four solar proton events observed by Explorers XII and XIV in 1961 and 1962 are discussed. These events are directly associated with solar activity and, in three cases, are followed either by secondary events delayed about 2 days or by recurrent events on succeeding solar rotations. It is shown that the rate of propagation of solar protons in some of these primary events is linearly dependent on particle velocity and that this dependence makes it possible to separate the source characteristics from the propagation effects. In each event which shows this velocity dependence the propagation curves of all observed energies agree and reach maximum intensity at a time much greater than the rectilinear travel time. These results lead immediately to the conclusions that propagation involves an important degree of scattering and that the degree of scattering is independent of energy over the observed range of 1.4 to $500 \mathrm{mev}$. The energy spectrum at the time of escape from the sun, the "source spectrum," is determined in each event which shows this velocity dependence and is described by a power law in kinetic energy from a few mev to several hundreds of mev. A striking feature of all events is the existence of periodic fluctuations in intensity, which are simultaneous at all energies, having a period of from 1 . to 1.5 hours depending on the event. This result indicates a local origin, and it is suggested that the fluctuations reflect processes occurring in the region between the magnetosphere and the earth's shock front.

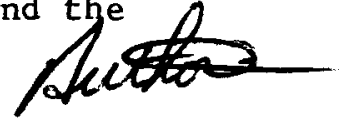

* Now at D.S.I.R. Radio Research Station, Slough, Bucks, England.

** Now at Physical Research Laboratory, Navrangpura, Ahmedabad, India. 


\section{INTRODUCTION}

During the last solar cycle it has become apparent that large solar flares are often accompanied by the acceleration of protons and other nuclei to energies sometimes exceeding tens of bev. Over a five-year period centered around the last solar maximum about sixty of these events were observed. Solar-proton events have been studied by many techniques. Ionization chambers, $\mathrm{mu}-\mathrm{mes}$ on telescopes, and neutron monitors at sea level provide indirect information at energies above a few bev. With high-altitude balloons, measurements down to about $80 \mathrm{mev}$ are possible. Rocket measurements have no inherent low-energy threshold but are limited to brief samples of an event. Studies by radio techniques of the ionization produced in the D layer are sensitive to energies down to about $10 \mathrm{mev}$ but have little energy resolution. Satellite studies can combine the advantages of the other techniques: they can provide almost continuous coverage with good energy and time resolution and with no inherent energy threshold, yielding a higher probability of detection of solar-proton events than can be provided by the other methods. Finally, a satellite with a sufficiently eccentric orbit can provide measurements of solar protons free of interaction with the earth's magnetic field and free of disturbances by the earth's trapped radiation.

The satellites Explorer XII and Explorer XIV carried cosmic-ray instruments with response for solar protons of energy as 1 ow as $1.4 \mathrm{mev}$. They were active from 16 August to 5 December 1961 and from 2 October 1962 to 1 August 1963, respectively. We discuss here several findings resulting from the study of the 
major solar proton events observed with these sate1lites during 1961 and 1962. Anomalous features of certain other events will be treated in later papers.

\section{THE APPARATUS}

Explorers XII and XIV were essentially identical satellites with similar orbits. The apogee of Explorer XII was about 13 earth radii and that of Explorer XIV about 16 earth radii; thus, for more than half the time in each orbit they were beyond the outer edge of the magnetosphere which varied from about 7 to over 12 earth radii. Gaps about 8 hours wide appear in the data as a result of discarding the observations made during the passes of the satellite through the magnetosphere. Various characteristics of the satellites are 1 isted in Table $I$.

\begin{tabular}{|c|c|c|}
\hline Parameter & Explorer XII & Explorer XIV \\
\hline Dates of Operation & 16 Aug. to 5 Dec. 1961 & 2 Oct. 1962 to 1 Aug. 1963 \\
\hline Orbital Period & 26.5 hours & 36.5 hours \\
\hline Apogee, Geocentric & $83,600 \mathrm{~km}$. & $104,800 \mathrm{~km}$. \\
\hline Initial G. Perigee & $6,700 \mathrm{~km}$ & $6,700 \mathrm{~km}$ \\
\hline $\begin{array}{l}\text { Sun-apogee angle } \\
\text { from earth }\end{array}$ & $\begin{array}{l}\approx 0^{\circ} \mathrm{min} . \text { to } \\
\approx 110^{\circ} \mathrm{max} .\end{array}$ & $\begin{array}{l}\approx 70^{\circ} \text { to } 180^{\circ} \\
\text { and back to } \approx 0^{\circ}\end{array}$ \\
\hline Initial Spin Period & 2.2 seconds & 5. seconds \\
\hline $\begin{array}{l}\text { Direction of Spin } \\
\text { Axis }\end{array}$ & $\begin{array}{l}+47^{\circ} \text { right ascension } \\
-27^{\circ} \text { declination }\end{array}$ & $\begin{array}{l}\text { (Uncertain due to } \\
\text { precession) }\end{array}$ \\
\hline
\end{tabular}


The detectors used for solar proton measurements in Explorers XII and XIV provided differential energy measurements from $1.4 \mathrm{mev}$ to $500 \mathrm{mev}$ and an integral measurement at about $600 \mathrm{mev}$. There were three detectors: a scintillation-counter telescope, a geiger-counter telescope and a single scintillation counter. The scintillation-counter telescope with a 32-channel pulse-height analyzer provided the differential-energy measurements for energies above $50 \mathrm{mev}$ and the integral measurement at $600 \mathrm{mev}$. The geigercounter telescope used in the single mode and in the coincidence mode gave integral measurements at $30 \mathrm{mev}$ and $100 \mathrm{mev}$. Low-energy measurements were made with the single scintillation counter and an 8-channel integral pulseheight analyzer. The axis of the geiger-counter telescope was oriented parallel to the satellite's spin axis and the other detectors were normal to it. A summary of the dynamic range and resolution of each of the detectors is given in Table II. Corrections were applied to all the data for particles penetrating the detector shielding and for particles clipping the edges of the scintillators; only when these corrections were small were the data used for analysis. The detectors are described more fully in a previous publication (Bryant et al., $1962)$ 


\begin{tabular}{|c|c|c|c|}
\hline Detector & Plastic Scintillator Telescope & Geiger Counter Telescope & CsI Crystal \\
\hline $\begin{array}{l}\text { Proton } \\
\text { Energy Range }\end{array}$ & $>55 \mathrm{mev}$ & $>30 \mathrm{mev}$ & 1.4 to $22 \mathrm{mey}$ \\
\hline $\begin{array}{l}\text { Information } \\
\text { Recorded }\end{array}$ & 32 differential channels & $\begin{array}{ll}\text { Coincidence } & \text { Single } \\
\text { mode: } & \text { mode: }\end{array}$ & $\begin{array}{l}8 \text { integra1 } \\
\text { levels }\end{array}$ \\
\hline $\begin{array}{l}\text { Energy } \\
\text { Intervals } \\
\text { Processed }\end{array}$ & $\begin{array}{rr}\text { Energy Interval; } & \text { Mean Energies } \\
55 \text { to } 118 & 87 \mathrm{mev} \\
118 \text { to } 150 & 135 \mathrm{mev} \\
150 \text { to } 200 & 175 \mathrm{mev} \\
200 \text { to } 255 & 228 \mathrm{mev} \\
255 \text { to } 335 & 295 \mathrm{mev} \\
335 \text { to } 500 & 418 \mathrm{mev} \\
& \text { (above } 600 \mathrm{mev} \text { ) }\end{array}$ & $\begin{array}{l}\text { Energy Lower Limits } \\
\approx 30 \mathrm{mev} \quad \approx 100 \text { mev } \\
\text { (sensitivity is a } \\
\text { function of energy) }\end{array}$ & $\begin{array}{r}\text { Mean Energies } \\
2.2 \mathrm{mev} \\
3.8 \mathrm{mev} \\
5.7 \mathrm{mev} \\
7.9 \mathrm{mev} \\
14.5 \mathrm{mev}\end{array}$ \\
\hline $\begin{array}{l}\text { Geometric } \\
\text { Factor } \\
\left(\mathrm{cm}^{2} \text { ster) }\right.\end{array}$ & $\begin{array}{l}13.4 \\
\text { (Corrections take into account } \\
\text { with energy) }\end{array}$ & $\begin{array}{cc}150 . & 12.7 \\
(\text { at } 100 \mathrm{mev})\end{array}$ & 2.85 \\
\hline $\begin{array}{c}\text { Time } \\
\text { Resolution }\end{array}$ & $\begin{array}{c}5 \text { minutes of storage each } 7 \\
\text { minutes }\end{array}$ & $\begin{array}{l}1.6 \text { seconds of storage } \\
\text { each mode during } 5 \text { out } \\
\text { of } 7 \text { minutes }\end{array}$ & $\begin{array}{l}1.6 \text { seconds } \\
\text { of storage } \\
\text { each level } \\
\text { for } 5 \text { out } \\
\text { of } 7 \text { minutes }\end{array}$ \\
\hline $\begin{array}{l}\text { Direction } \\
\text { of Detector } \\
\text { Axis }\end{array}$ & Normal to spin axis & Parallel to spin axis & $\begin{array}{l}\text { Normal to } \\
\text { spin axis }\end{array}$ \\
\hline
\end{tabular}

TABLE II

DETECTOR CHARACTERISTICS 
III. THE EVENTS

In this paper we confine our attention to the solar proton events following the flares of 10 September, 28 September, and 10 November 1961 and the flare of 23 October 1962. Some details of these flares and the plage regions producing them are given in Table III. The table also contains comments on the flares (H. Prince and R. Hedeman, private communication, 1963) and on the accompanying type IV radio emission (A. Maxwel1, J. Warwick, private communications, 1963). Some of the flares were followed by delayed effects and these are also 1isted. Effects delayed by the prompt 2-day sun-to-earth transit of enhanced plasma and effects delayed by the rotation of the sun (27-day recurrent events) are 1 isted separately.

A calendar of all the solar particle events observed during the intervals discussed here is shown in Figure 1, in which the occurrence of primary events and delayed effects are plotted against the cosmic-ray intensity measured by a sea-leve1 neutron monitor (courtesy of H. Carmichael, private communication, 1962). Examination of the data showed no evidence either for any other smaller discrete events or for any separate, continuous increases over the general background level. The intensity of one energy interval of protons, centered at $87 \mathrm{mev}$, is plotted for al1 five primary events in Figure 2, indicating the widely varying behavior of the events. The event of 7 September 1961 , which does not lend itself to obvious correlation to any known flare, will be discussed in a later paper. 
TABLE III

DETAILS OF THE FLARES

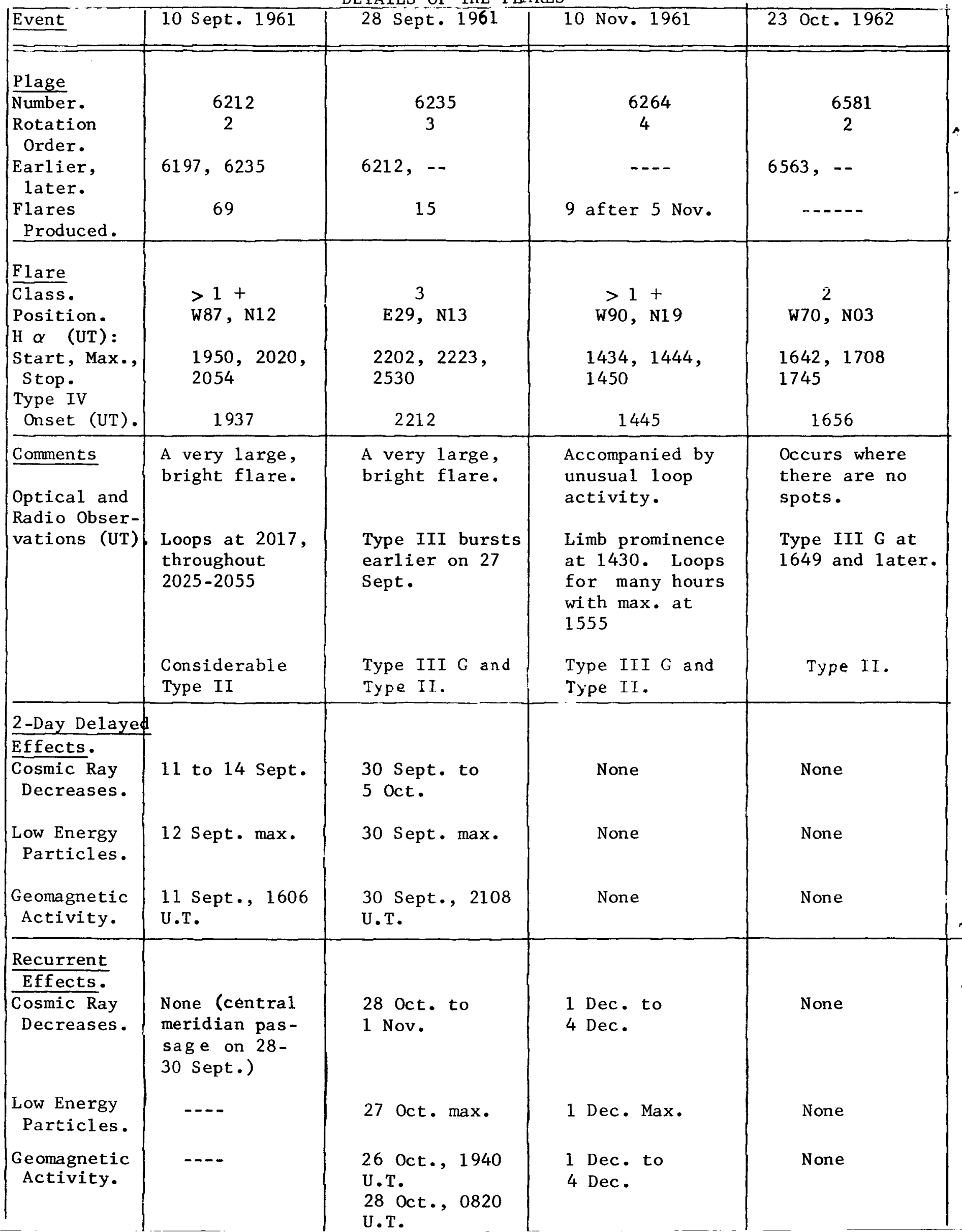


IV. THE EVENT OF 28 SEPTEMBER 1961

The solar proton event of 28 September 1961 was initiated by a class 3 flare $29^{\circ}$ east of central meridian. Table III shows the pertinent solar and geophysical data. Some aspects of the event were considered in an earlier paper (Bryant et al., 1962).

Intensity vs. time profiles of various energy components of the event are shown in Figure 3. Time is measured in hours from $2208 \mathrm{UT}$, the time of emission at the sun of a brief $x$-ray burst observed by Anderson and Winckler (1962) from 2216 to 2217 UT. For the first few hours points are plotted at intervals of about 7 minutes, and later points are hourly averages. The energy parameter is the mean energy of each interval as outlined in Table II. It is clear that the time taken to reach maximum intensity increases with decreasing energy. The data are less complete at the lower energies because at first the correction due to high-energy particles which penetrate the shielding of the low-energy particle detector is large, and because the satellite entered the trapped-radiation zone soon after the first effect became small.

There is a departure from a smooth decay at about 48 hours. At that time an increase took place in the intensity of low-energy particles which was associated with the arrival of a solar plasma stream that produced a suddencommencement geomagnetic storm and a Forbush decrease. This occurrence is described in detail in the earlier paper, but for the sake of completeness it is briefly described here. The details of this increase, omitted in Figure 3, are shown for some energy intervals in Figure 4 on a linear time scale. Late on 30 September there is a sudden increase in intensity which 
is more marked at lower energies. The increase starts at about $1930 \mathrm{UT}$ just before the sudden commencement of a magnetic storm at $2108 \mathrm{UT}$. The maximum intensity of particles of energy above $3 \mathrm{mev}$ during the increase is more than ten times that attained during the main solar-proton event. Axford and Reid (1962) reported riometer observations of this burst of lowenergy protons and had previously observed a similar event on 10 February 1958 (Reid and Axford, 1962). Other events of this kind have been reported by Charakhchyan et a1. (1963).

After a full rotation of the sun, when the plage region responsible for the flare of 28 September was again close to central meridian, there was another burst of low-energy protons. This time the increase was much smaller. Figure 5, which is a plot of the total intensity above 3 mev on a further-compressed linear time scale, shows the full sequence of events. This recurrent event was also associated with enhanced solar plasma responsible for a recurrent geomagnetic storm and a recurrent Forbush decrease. We have put forward this event and a similar one following the 10 November 1961 flare as new evidence for the existence of long-lived solar streams (Bryant et al., 1963).

\section{a) Velocity Dependence}

The energy dependence of the rate of rise of intensity suggests that the propagation of the particles is a velocity-dependent process. A striking linear dependence on velocity is revealed by the following analysis.

We assume that a11 particles were accelerated at the same time or, more strictly, that they were all accelerated within a time interval short 
compared with the interval between acceleration and observation. We may then determine the distance a particle has travelled between acceleration and observation by taking the product of particle velocity and time from the beginning of the event. The time used here for the beginning of the event is $2208 \mathrm{UT}$, the time of the $\mathrm{x}$-ray burst at the sun. We then convert the intensity vs. time profile to an intensity vs. distance profile. The intensity vs. distance profile is effectively a distribution in distance travelled. This distribution is a property of the medium through which the particles have travelled. Figure 6 shows the result of this treatment in which the distance travelled is measured in astronomical units. In constructing this figure the intensities of each component have been scaled to give the best fit to a common curve. The physical meaning of this normalization will be examined further below.

We note from Figure 6 that all components lie very closely on a common curve, apart from small-scale deviations to be discussed later as a separate topic. The fact that we have essentially a common curve shows that particles of all energies have travelled a given path length with equal probability; this is true for al1 path lengths to the extent that the various components of Figure 6 do lie on a common curve. We note that the distance travelled by most particles is many astronomical units, which indicates that propagation involved an important degree of scattering. Further, the degree of scattering is not a function of energy over the observed range. This suggests that the mode of propagation is a diffusion-like process and that energy-dependent processes, such as drift across magnetic field lines, play a minor role. In fact, it has been shown that the equation for simple diffusion describes 
the propagation curve of this particular event through its maximum (Bryant et a1., 1962). It fails to do so, though, during both the early anisotropic phase and the later stages where boundary conditions are important.

\section{b) Source Spectrum}

We now discuss the physical meaning of the scaling factors used to construct Figure 6. Consider the relative intensity of any two components of the event. We have recorded the intensities not as a function of time but as a function of distance travelled, and found that the relative intensity is essentially constant over a range from 2 to more than 100 astronomical units. There is nothing to suggest that an extrapolation back to zero distance is invalid. The relative intensity of two components at zero distance is, by definition, a measure of the shape of the source spectrum.

The scaling factors used to produce Figure 6 provide these relative intensities and Figure 7 shows the source spectrum obtained directly from them. The source spectra of two other events analyzed in a similar way are also shown. The ordinate of Figure 7 is arbitrarily chosen to show the maximum intensity reached at the earth. The differential intensities shown are proportional to the absolute differential intensities of protons produced at the sun and retain, therefore, the same spectral form, but the constant of proportionality which depends on the geometry of propagation is unknown. The spectrum is well represented by a power law in kinetic energy with a slope of about -1.7 .

We note that, apart from small-scale fluctuations, this event is completely described by two graphs: the source spectrum of Figure 7 and the distribution of path length during propagation of Figure 6 . 
V. THE EVENT OF 23 OCTOBER 1962

The event of 23 October 1962 is the smallest discussed here and probably the lowest-intensity primary solar-proton event studied to date. It was initiated by a class 2 flare occurring $70^{\circ}$ west of central meridian; further details of the flare and associated phenomena are listed in Table III. The event was also observed by the cosmic-ray equipment on Mariner II (H. Anderson and V. Neher, J. Van Allen, private communications, 1963).

Figure 8 shows the intensity vs. time profiles of various components of the event. We see that again the higher-energy components reach a maximum earlier than the 1 ower-energy components. The intensity vs. time profiles, corrected for particle velocity and superimposed in the same way as for the event of 28 September 1961, are shown in Figure 9. We find a good fit to a common curve so again the observation that higher-energy particles arrive earlier is explained quantitatively as a dispersion effect. The source spectrum obtained from the relative normalization used to construct Figure 9 is shown in Figure 7 . We note that again it is well represented by a power law in kinetic energy. The slope of the spectrum in this case is about -2.3

VI. THE EVENT OF 10 NOVEMBER 1961

The solar proton event of 10 November 1961 was one of two events observed by Explorer XII resulting from flares on the west limb of the sun (Table III). A long-1ived solar stream emanating from the flare region produced a recurrent event 21 days later on 1 December (Bryant et al., 1963). 
Intensity vs. time profiles for this event are shown in Figure 10. A feature of these curves is a sudden drop in intensity at 1.3 hours (1546 UT). It occurs in all components that show a measurable intensity at this time. A second drop occurs at 3 hours (1730 UT). As we shall mention again in Section VII, these fluctuations are very likely unusually large cases of a feature common to all four primary events discussed here, namely a periodic intensity fluctuation with a period of about one hour. However, since these particular changes are so unusually large and sharp they may be due to some other cause. The flare producing this event was accompanied by unusual loop activity, so it may not be by chance that the solar-proton intensity exhibits unusual behavior. We are unable to link any specific happening at the sun with the changes taking place near the earth but we are able to deduce when it should occur. Figure 11 shows the integral intensities above $60 \mathrm{mev}$ and above 200 mev plotted in a linear scale to show how drastically the behavior changed. The data are consistent with the breakdown having occurred simultaneously at all energies, but the time resolution of the measurements would permit a 15 minute dispersion and so is consistent with the dispersion to be expected from the velocity difference acting over a distance of 1 astronomical unit. The breakdown occurs in the $87 \mathrm{mev}$ component somewhere between 1546 and 1556 hours. Since 87 mev particles take 20 minutes to travel 1 a.u., the first ones to bring information of a change would have left the sun between 1526 and 1536 if they travelled in a straight line, but (more likely) between 1521 and 1531 if they travelled along a curved path defined by the interplanetary magnetic field. Optical or radio information would reach the earth 8 minutes later, somewhere between 1529 and 1539. Though the flare showed great activity (see Table III for comments) we have found no evidence for any drastic 
change of conditions in this period.

a) Velocity Dependence and Source Spectra

The complicated structure of this event defies a description in terms of velocity dependence alone. As will be shown below, there are occasions on which a velocity dependence is revealed. Figures $12 \mathrm{a}$ and $12 \mathrm{~b}$ show velocity-compensated intensity-distance profiles for the higher and lower energies taken separately. The higher-energy components are velocity dependent before the sudden drop in intensity at 1.3 hours, and the lower-energy components are velocity dependent throughout the event. The fact that the lower-energy components do not show a measurable intensity until after the breakdown of velocity dependence at higher energy introduces a further uncertainty in interpretation.

Two source spectra are shown for this event in Figure 7 , one for the higher and one for the lower energies. Since the velocity-compensated intensity vs. time profiles are different, the relative normalization of the two sections of the spectrum is not meaningful. The normalization for each section of the spectrum is arbitrarily chosen to be the maximum intensity reached at the earth. It may be that the common fit of the low-energy intensity curves is not significant, since the deviation from a common propagation envelope increases as energy decreases among the high-energy groups.

b) Time Dependence

Although this event shows a velocity dependence that holds very well at certain times, it cannot be completely described by a single source spectrum and single propagation curve. Further, there are two sudden 
intensity drops that occur over a wide energy range with no dispersion. They almost certainly result from sudden changes in the propagation medium which probably take place near the earth but, from the above discussion, which may also take place at the sun. Other effects of this kind are described in the next section and discussed more fully in section VIII.

VII. THE EVENT OF 10 SEPTEMBER 1961

The event of 10 September 1961 was initiated by a flare on the west limb of the sun. The intensity vs. time profiles of Figure 13 show that the event was dominated by intensity changes occurring simultaneously at all energies. Only gross features, such as the fact that the lower-energy components reach maximum intensity later than the higher-energy components, can be attributed to dispersion. There are no systematic changes of behavior with energy and consequently no quantitative fit to velocity dependence. A linear plot of three sample components (Figure 14) typifies the irregular behavior of the event. The high intensity reached by this and lower-energy components early on 12 September, about 1 day after the flare, is probably due to the arrival of enhanced solar plasma that produced a small cosmic-ray decrease at about that time following geomagnetic activity at 1106 UT on 11 September. This increase is like the delayed arrival of low-energy particles seen about 48 hours after the 28 September 1961 event, but in this case there is a much slower onset no doubt having to do with the west limb location of the flare.

\section{a) Fluctuations}

Superimposed on the large-scale features of the intensity vs. time profiles are a series of fluctuations which are nearly periodic, with the same frequency and phase at all energies. They take place in all these events, but are most 
clearly marked in the event of 10 September 1961. Figure 15, which shows the integral intensity vs. time profiles on a linear scale for the 5.7 -mev and the 30-mev components of this event, illustrates these fluctuations. To show the periodic fluctuations more clearly, the lower-frequency components have been removed by subtracting the running mean of one period length; the result for several energy components is shown in the lower half of the figure. Fluctuations of this kind occur to some extent in all events: Figure 16, in which the relative time scale is in units of hours, shows the fluctuation of the $87-$ mev component for all events. The sudden drops in intensity near the beginning of the 10 November 1961 event have the same frequency and phase as the other fluctuations, so it appears that they may not be isolated happenings caused directly by activity at the sun but the first two members of a series of fluctuations, although they were unusually large and very sharp. The amplitude of the fluctuations depends little on energy in any of the events. Table IV summarizes the periods and amplitudes of the fluctuations.

\section{DISCUSSION}

The following general statements can be made summarizing the results presented above.

(1) Solar proton events observed in the 2- to 600-mev energy region fall into at least three categories: the primary events have maximum intensity soon after the parent flare; these are sometimes followed about two days later by secondary events that occur with the arrival of the solar plasma; they also are occasionally followed by recurrent events that do not immediately follow solar activity but occur when the parent plage region passes central meridian on successive 27-day intervals. 
(2) The intensity vs. time profiles often show a linear velocity dependence. In two of the events under discussion (28 September 1961 and 23 October 1962) this velocity dependence lasts throughout the events. Exceptions occur in the event of 10 November 1961, which shows some early departure from this behavior and in the event of 10 September 1961, which shows no quantitative agreement with velocity dependence.

(3) All events show a series of periodic intensity fluctuations with a period of from 1 . to 1.5 hours. These occur without dispersion.

If we convert those intensity vs. time profiles which show a quantitative, linear velocity dependence to probability vs. distance profiles, we are led directly to the following conclusions:

(4) Properties of the propagation medium and properties of the source can be studied separately and, in particular, the shape of the energy spectrum at the source can be obtained.

(5) Propagation involves an important degree of scattering (since the most likely distance travelled is about 10 astronomical units).

(6) The degree of scattering is independent of particle rigidity in the region below 1.5 bv (since all the propagation curves are the same).

\section{a) Velocity Dependence}

The analysis described in section IV, showing the velocity dependence of these events, has constituted a necessary but insufficient test. For example, we might have found an even closer fit had we compensated the intensity vs. time profiles by velocity raised to the power $n$, where $n$ is close to but not equal to 1 . We now show that the best value of $n$ is indeed 1 . To find the best value of $n$, the various intensity vs. time profiles were shifted 
horizontally and vertically to give the best fit to a chosen reference curve. No account was taken of particle velocity at this stage. The factors by which the curves were horizontally shifted were then plotted against particle velocity. The result is shown in Figure $17 \mathrm{a}$ in which, for comparison, lines of slope unity have been drawn through the points. The normalizations are arbitrary and depend on the curves chosen for reference. We find that the slopes of all lines are close to unity, the mean value being $1.0 \pm 0.1$. The energy intervals over which this test could be performed are implied by the figure and are indicated in Table IV. The various parts of Figure 17a have been superimposed in Figure 17b, where, as in 17a, a line of slope unity has been drawn through the points for comparison. (The energy range studied here does not extend sufficiently far into the relativistic region to permit a test of rigidity dependence as opposed to simple velocity dependence.)

Further evidence of velocity-dependent propagation in solar-proton events was provided by a comparison of alpha-particle and proton intensities during the events of 12 November 1960 and 15 November 1960 by Biswas et al. (1962, 1963). Several rockets carrying nuclear emulsions were fired into these events, and the relative intensity of alpha particles and protons having the same velocity was found to be the same at certain times even though the energy spectra of protons and alpha particles were changing with time.

\section{b) The Source Spectrum}

We note from Figure 7 that a given source spectrum is well represented by a power law in kinetic energy, although the event of 10 November 1961 is an exception in that two power-1aw spectra are required. The 28 September 1961 spectrum is remarkable in that it fits a power law over a dynamic range of nearly three decades in energy. The fact that there is no deviation from 
this smooth, simple spectral representation, even at the low-energy portion of this source spectrum, prompts us to put forward the argument, based purely on aesthetic grounds, that the amount of matter traversed by the solar protons after acceleration was less than the range of 1 mev proton, that is, about 1 milligram $\mathrm{cm}^{-2}$. If we consider a beam of particles penetrating an absorber, we find the emerging spectrum very much depleted in particles with energies near that required to just penetrate the absorber. There is consequently a rapid change of slope at low energies. It seems unlikely, therefore, that an excess production of lower-energy protons would occur in such a manner as to exactly compensate their absorption in an amount of material greater than their range resulting in so simple a form of source spectrum.

We can draw no conclusions here about the solar-proton acceleration process from the shape of the source spectra, since there probably are a number of processes that would produce the observed spectra. Table IV gives the slopes of the spectra observed in the events under discussion.

c) The Propagation Medium

For the purpose of this discussion we define the propagation medium to be the medium through which solar protons travel after acceleration and escape from the acceleration mechanism. Let us now consider where the scattering takes place.

Meyer, Parker and Simpson (1956) suggested that during the 23 February 1956 event scattering took place in interplanetary space beyond the earth's orbit. Parker (1963) has discussed a mechanism of simple diffusion throughout interplanetary space; E. Roelof (private communication, 1963) has found that 
scattering by magnetic fields in interplanetary space that are irregular in space and/or time would produce a degree of scattering that is a function of particle rigidity. McCracken (1962) introduced the idea of smal1-angle scattering caused by irregularities in an otherwise quasi-radial interplanetary field to account for the onset of isotropy in several solar proton events. Anderson et al. (1959) and Gold (1962) have suggested that the processes of scattering and drift in the strong magnetic fields near the sun must play an important part in solar-proton propagation. Bryant et al. (1962) and Hoffman and Winckler (1963) found that the intensity vs. time profiles of several solar proton events are well represented by those to be expected from a process of simple diffusion in interplanetary space.

The propagation curves of the three velocity-dependent events are compared in Figure 18. Apart from that of the low-energy component of 10 November 1961 , the curves are geometrically similar to a remarkable degree. It is meaningful, therefore, to associate with each of the curves a parameter describing the rate of propagation of the event. A convenient parameter, though somewhat poorly defined, is the most probable distance travelled --that is, the distance travelled by the particles arriving at maximum intensity. These distances are listed in Table IV. We notice that the ordering of the propagation geometry in the three events correlates either with the phase of the solar cycle or with the displacement in longitude of the flare from about 70 degrees west, the origin of the earth-intercepting gardenhose line. For example, the slowest event is that from a flare at 29 degrees east; it is also the first in time. No significance is claimed for either correlation on the basis of such a small number of events. 
We have mentioned that the intensity curves which fit a common propagation curve in a given event also fit the equation for simple diffusion. The resulting effective mean free paths and other parameters are listed in Table IV. In spite of this fit, it is difficult to see how solar protons could undergo a process of simple diffusion in interplanetary space especially since the garden-hose magnetic field has been observed to play an important part in guiding solar protons away from the sun. Another difficulty is that the observed velocity independence of the scattering implies that the scattering takes place at discrete scattering centers, rather than as a continuous process, as would otherwise seem more reasonable. The finding that the degree of scattering is independent of rigidity, combined with Roelof's result for continuous scattering in irregular magnetic fields, rules out such a mechanism at least for the events under discussion.

In order to reconcile a diffusive propagation with the previously observed guiding by the garden-hose interplanetary field, we consider a model in which scattering occurs near the sun and the particles escape to the earth after diffusing from the flare to the foot of a line of force providing direct access to the earth (Gold, 1959). We meet at once with two difficulties. Firstly, particle drift produced by gradients and curvatures in any general field near the sun would lead to a rigiditydependent propagation. Secondly, there must be a certain amount of scattering in interplanetary space to account for the onset of isotropy (McCracken, 1962). Since any pitch-angle scattering would in general be accompanied by a change of guiding center there would be some motion across the lines of force. The 
question then arises of the relative importance of motion along the lines of force and motion across them. We have then reached a process of anisotropic diffusion in interplanetary space instead of one of simple isotropic diffusion. Anisotropic diffusion conceivably could account for the observations, but there is no present theoretical treatment against which to test the data.

\section{d) The Stability of the Propagation Medium}

One important consequence of a velocity-dependent propagation is that low-energy protons travel through the propagation medium later than the highenergy protons and yet suffer the same degree of scattering. The average properties of the propagation medium can, therefore, remain constant for at least two days. It is important to remember, though, that changes must have been occurring in the magnetic field structure during the period of propagation. During the event of 28 September 1961, for example, there was an enhanced solar plasma moving out from the sun. Where the solar protons are isotropic in interplanetary space, even gross changes in magnetic field structure can produce only small effects if they take place many mean free paths from the point of observation. Only changes in magnetic field structure occurring near the satellite could strongly influence the measured intensity. We suggest that the character of the 10 September 1961 event was largely determined by such effects, dominating the intensity vs. time character more than the dispersion that otherwise should have been present. One can only speculate at this stage on the details of the mechanism producing time-dependent effects. 
e) Local Disturbances of the Propagation Medium

As was mentioned above, the periodic intensity fluctuations are almost certainly a result of magnetic field structure in the region of interplanetary space near the earth. The fluctuations could result from, for example, adjacent regions of strong field and weak field constituting a trapping region or "magnetic bottle." Such regions would exclude some incident particles, thereby lowering the particle density in the region of the weaker field. Such regions might eventually become filled as a result of scattering from magnetic irregularities and, in fact, the magnitude of the fluctuation does decrease with time. This mechanism does not account for the regularity of the fluctuation unless there is postulated a characteristic wave motion of interplanetary plasma. The wave motion is not a property of all interplanetary plasma but is confined to the region between the magnetosphere and the quasistationary bow wave postulated by Axford (1963) and others. They pointed out that interaction between the solar wind and the magnetosphere should create a bow wave standing several earth radii from the magnetosphere on the sunlit side of the earth; this phenomenon was recently observed with a plasma detector (Bridge et al., 1964) and a magnetometer (Ness et a1., 1964) on Explorer XVIII. A11 solar-proton measurements reported here were made outside the magnetosphere but behind this bow wave. It is possible, therefore, that the modulation of solar-proton intensity was confined to this region alone: regular structure in this region may be due directly to regular structure in the solar wind or it may be a natural frequency of the region.

Fluctuations with a period of approximately one hour were noted in the Explorer X magnetic field and plasma data (Heppner et a1., 1963; Bonetti et al.., 
1963) where they were interpreted as a result of the passage of the space probe in and out of the pulsating boundary of the magnetosphere. Mathews et a1. (1961) noted fluctuations with a period of 1.25 hours in neutron monitor records of the event of 12 November 1960. They suggested that these fluctuations were a direct result of pulsating decreases in the horizontal component of the earth's field measured at the equator. Winckler et al. (1961) also observed similar fluctuations in balloon measurements of solarproton intensity. All of these forms of fluctuation may have been due to the same cause, namely, either a regular structure in the interplanetary plasma density or a natural frequency of the region between the bow wave and the magnetosphere.

TABLE IV

SOLAR-PROTON EVENT CHARACTERISTICS

\begin{tabular}{|c|c|c|c|c|c|}
\hline Event & 10 Sept. 1961 & 28 Sept. 1961 & $\begin{array}{r}10 \text { Nov } \\
\text { Low Energy }\end{array}$ & $\begin{array}{l}1961 \\
\text { High Energy }\end{array}$ & 23 oct. 1962 \\
\hline $\begin{array}{l}\text { Energy Range } \\
\text { of Velocity } \\
\text { Dependence } \\
\text { (mev) }\end{array}$ & $\begin{array}{c}\text { (none } \\
\text { detected) }\end{array}$ & 1.4 to 500 & 1.4 to 22 & 55 to 500 & 4 to 330 \\
\hline $\begin{array}{l}\text { Exponent of } \\
\text { Source } \\
\text { Spectrum }\end{array}$ & $-\cdots-\cdots$ & -1.7 & -1.5 & -3.5 & -2.3 \\
\hline $\begin{array}{l}\text { Scale of } \\
\text { Source Spectrum } \\
\text { at Max. Intens. } \\
\left(\mathrm{cm}^{2} \mathrm{sec} \text { ster }\right.\end{array}$ & mev) $)^{-1}$ & $1.6 \times 10^{6}$ & $1.4 \times 10^{6}$ & 7. $\times 10^{9}$ & 4. $\times 10^{5}$ \\
\hline $\begin{array}{l}\text { Most Probable } \\
\text { Distance } \\
(\text { a. u.) }\end{array}$ & --- & 12 & 8 & 9 & 8 \\
\hline $\begin{array}{l}\text { M.F.P. (a. u.) } \\
\text { from Diffusion } \\
\text { Theory }\end{array}$ & ---- & 0.04 & 0.06 & 0.055 & 0.06 \\
\hline $\begin{array}{l}\text { Fluctuation } \\
\text { Period (hours) }\end{array}$ & 1.4 & 1.3 & $\begin{array}{l}\text { (none } \\
\text { detected) }\end{array}$ & 1.5 & 1.0 \\
\hline
\end{tabular}


REFERENCES

Anderson, K. A., Arnoldy, R., Hoffman, R., Peterson, L., and Winckler, J. R. 1959, J. Geophys. Res., 64, 1133 .

Anderson, K. A., and Winckler, J. R. 1962, J. Geophys. Res., 67, 4103 .

Axford, W. I., and Reid, G. C. 1962, J. Geophys. Res., 67, 1692.

Axford, W. I. 1962, J. Geophys. Res., 67, 3791 .

Biswas, S., Fichtel, C. E., and Guss, D. E. 1962, Phys. Rev., 128, 2756.

Biswas, S., Fichtel, C. E., Guss, D. E., and Waddington, C. J. 1963, J. Geophys. Res., 68, 3109.

Bonetti, A., Bridge, H. S., Lazarus, A. J., Rossi, B., and Scherb, F. 1963 , J. Geophys. Res., 68, 4017.

Bridge, H., Egidi, A., Lazarus, A., and Lyon, E. Explorer XVIII Symposium, Goddard, 1964.

Bryant, D. A., Cline, T. L., Desai, U. D., and McDonald, F. B. 1962, J. Geophys. Res., 67, 4983.

1963, Phys. Rev. Letters, 11, 144.

Charakhchyan, A. N., Tulinov, V. E., and Charakhchyan, T. N. 1961, Proc. Intern. Conf. Cosmic Rays and the Earth Storm, - Kyoto, 2, 365.

Gold, T. 1959, J. Geophys. Res., 64, 1665 .

1962, J. Phys. Soc. Japan, 17 (A -2), 600.

Heppner, J. P., Ness, N. F., Scearce, C. S., and Ski11man, T. L. 1963, J. Geophys. Res., 68,1 .

Hoffman, D. J., and Winckler, J. R. 1963, J. Geophys. Res., 68, 2067.

Mathews, T., Tambyahpillai, T., and Webber, W. R. 1961, Month1y Notices of the Royal Astron. Soc., 123, 97.

McCracken, K. G. 1962, J. Geophys. Res., 67, 423, 435 and 447.

Meyer, P., Parker, E. N., and Simpson, J. A. 1956, Phys. Rev., 104, 768. 
Ness, N., Scearce, C., and Skillman, T. Explorer XVIII Symposium, Goddard, 1964.

Parker, E. N. 1963, Interplanetary Dynamical Processes, (New York: Interscience Publishers).

Reid, G. C., and Axford, W. I. 1962, J. Geophys. Res., 67, 3590.

Winckler, J. R., Bhavsar, P. D., and Peterson, L. 1961, J. Geophys. Res., 66, 995. 
FIG. 1.-- Solar particle events observed with Explorers XII and XIV during 1961 and 1962. The occurrence of each primary solar proton event is indicated above the cosmic-ray neutron intensity plots with a flag which displays the solar longitude and classification of the parent flare and the existence of associated type IV radio bursts. Occurrences of both varieties of delayed proton events are also indicated; a plasma-associated event occurs with a delay of two days and a recurrent event takes place when a long-1ived solar streamer passes central meridian on a succeeding solar rotation.

FIG. 2.-- The intensities of $87-$ mev protons vs. time after the type IV emissions during the five primary solar proton events. The shapes of only two events, those of 28 September 1961 and 23 October 1962, are seen to be quantitatively similar, as monitored in this manner.

FIG. 3.- The differential intensities of solar protons during the 28 September 1961 event plotted against time after the $x$-ray burst at the sun. The data are interrupted when the satellite passed through the magnetosphere and when the delayed increase occurred on 30 September 1961.

FIG. 4. -- Representative proton intensities between 28 September and 7 October, showing the delayed intensity increase of predominately lowerenergy protons on 30 September 1961 . The energy spectra of these particles are relatively constant with time, unlike those of the velocity-ordered primary solar proton event, and their arrival times are essentially constant with energy, occurring at the time of arrival of the enhanced solar plasma, two days after the flare. 
FIG. 5. -- The intensity of protons of energy above 3 mev between 30 September and 28 October 1961. The delayed increase on 30 September is superposed on the primary solar-proton intensity decay and the recurrent event on 27 October follows the completely event-free intervening period.

FIG. 6. -- The intensity vs. time plots of Figure 3 converted to relative intensity vs. distance plots. The distance is computed for each energy component by taking the product of the corresponding particle velocity and time from the event; the intensities are scaled to give the best fit to a common propagation curve. This fit occurs over a dynamic range in energy of a few hundred and a velocity range of 14 , and over a time duration of several days.

FIG. 7. -- The source spectra of three solar proton events. The intensities plotted are arbitrarily chosen to show the maximum intensities reached at the earth; as explained in the text the relative scaling of the two sections of the spectrum of 10 November is not necessarily meaningful. In the case of the solar proton events which totally conform to a velocity-dependent behavior, such as those of 28 September 1961 and 23 October 1962, the source spectrum is the unique differential energy spectrum of the protons at the time of their escape from the sun; in each event the source spectrum is proportional to that shown here with a constant which depends in an unknown way on the geometry of propagation.

FIG. 8. -- The differential intensities of solar protons during the 23 October 1962 event plotted against time after the onset at the sun of type IV radiation. The behavior of this event is qualitatively similar to that of the early portion of the 28 September 1961 event. Due to the relatively low intensity of the event, 
the differential intensities of protons of energy above 300 mev could not be measured; the protons of energy below 5 mev did not arrive until after the satellite entered the mgnetosphere.

FIG. 9. -- The intensity vs. time plots of Figure 8 converted to a relative intensity vs. distance plots, scaled to give the best fit to a common propagation curve.

FIG. 10. -- The differential intensities of solar protons during the 10 November 1961 event plotted against time after the flare. A sudden drop in the intensities of the higher-energy protons occurs at 1.3 hours; this discontinuity in the slopes of the propagation curves occurs before the arrival of most of the lower-energy protons.

FIG. 11. -- The integral intensities of protons above $60 \mathrm{mev}$ and above 200 mev during the first few hours of the 10 November 1961 event, plotted on a linear scale. These components are chosen so as to display the maximum dispersion of the times of peak intensity; since measurements were made every 7 minutes, the data are consistent with the peak intensities having occurred simul taneously.

FIG. $12 \mathrm{a}$ and $12 \mathrm{~b} .--$ The intensity vs. time plots of Figure 10 converted to relative intensity vs. distance plots. Two groups are separately scaled to give the best fit to a common high-energy curve before the 1.3-hour peak, and to a co:mon low-energy curve after it. A unique high-energy propagation curve does not exist throughout the event, but appears to be approached asymptotically with increasing energy. A common low-energy curve is apparent, but it may relate more directly to a local modulation than to interplanetary propagation. 
FIG. 13. -- The differential intensities of solar protons during the 10 September 1961 event plotted against time after the flare. There is a qualitative velocity ordering for the first 10 hours; during this time the higher-energy protons tend to increase in intensity before the lower-energy protons do, but there is no quantitative fit to a velocity dependence. Later in this event the intensities of protons of all energies increase together in a manner qualitatively similar to that of 30 September; this occurrence is also accompanied by cosmic-ray and geomagnetic fluctuations associated with the arrival of enhanced solar plasma.

FIG. 14. -- A linear plot of the intensity of minimum-ionizing protons and of two lower-energy groups during the 10 September event, displaying both the irregular behavior of the intensity early in the event and the delayed increase on 11 September. These intensity variations dominate the pattern of this event.

FIG. 15. -- Linear plots vs. time of two integral proton intensities during the 10 September event; the deviations from the running means of intensity of these and two differential intensities are also shown in order to illustrate the relative fluctuations. A regular modulation appears having period and phase that are constant with energy; the times of minimum relative intensity are indicated to illustrate the approximate periodicity. Since there is no discernable velocity dispersion of this modulation within an event, these fluctuations are believed to have a local origin.

FIG. 16. -- The fluctuations of the 87 -mev component of the solar proton intensities of four solar proton events. The period of the fluctuation is 
$-31-$

constant within an event but varies from 1 . to 1.5 hours with the event.

FIG. $17 \mathrm{a}$ and $17 \mathrm{~b}$. -- The scaling factors by which the differential intensity vs. time plots of each event were shifted horizontally to give the optimum fit to a common curve. This effort was made to determine the nature of the dependency on velocity; comparison lines of slope 1 are used to indicate the closeness of fit to a linear velocity dependence.

FIG. 18. -- The propagation curves of the three velocity-dependent events. These curves represent distributions in the distance travelled by solar protons from release into the propagation medium to the point of observation. 


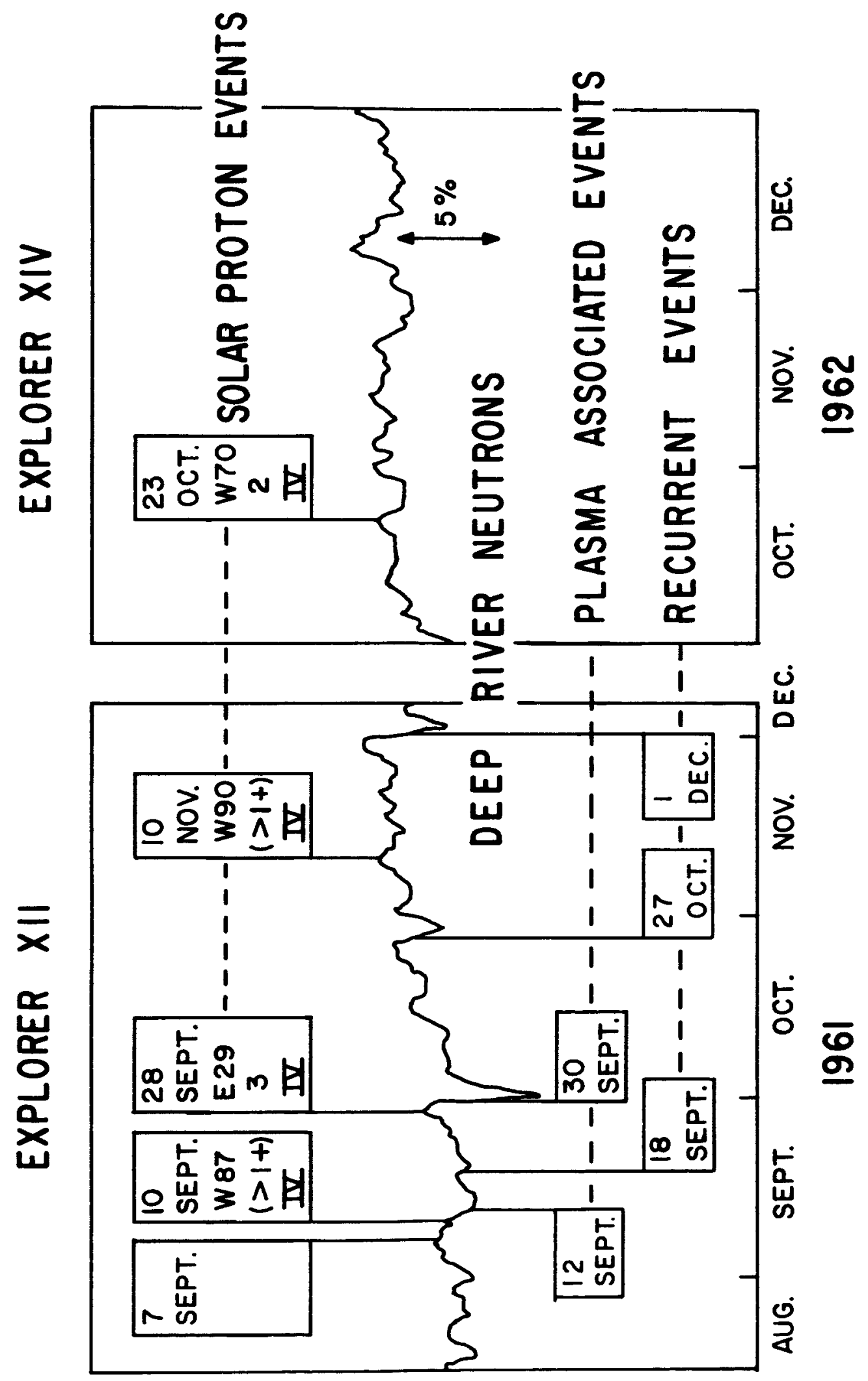

FIG. 1 


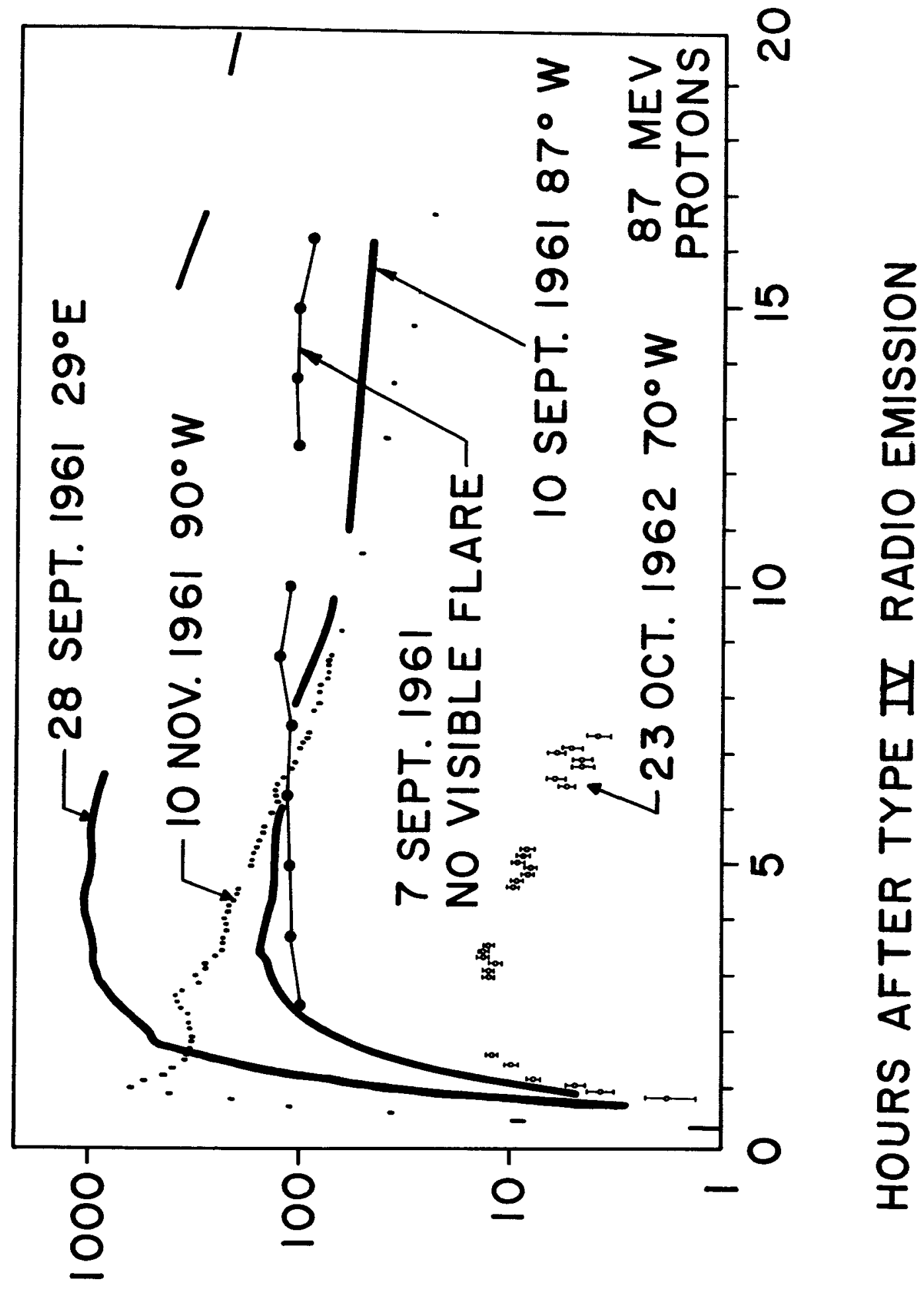

( $\triangle \exists W$ y $I S$ OJS $\left.{ }_{2} W / S N O \perp O Y d\right) ~ \wedge \perp I S N \exists \perp N I$ 


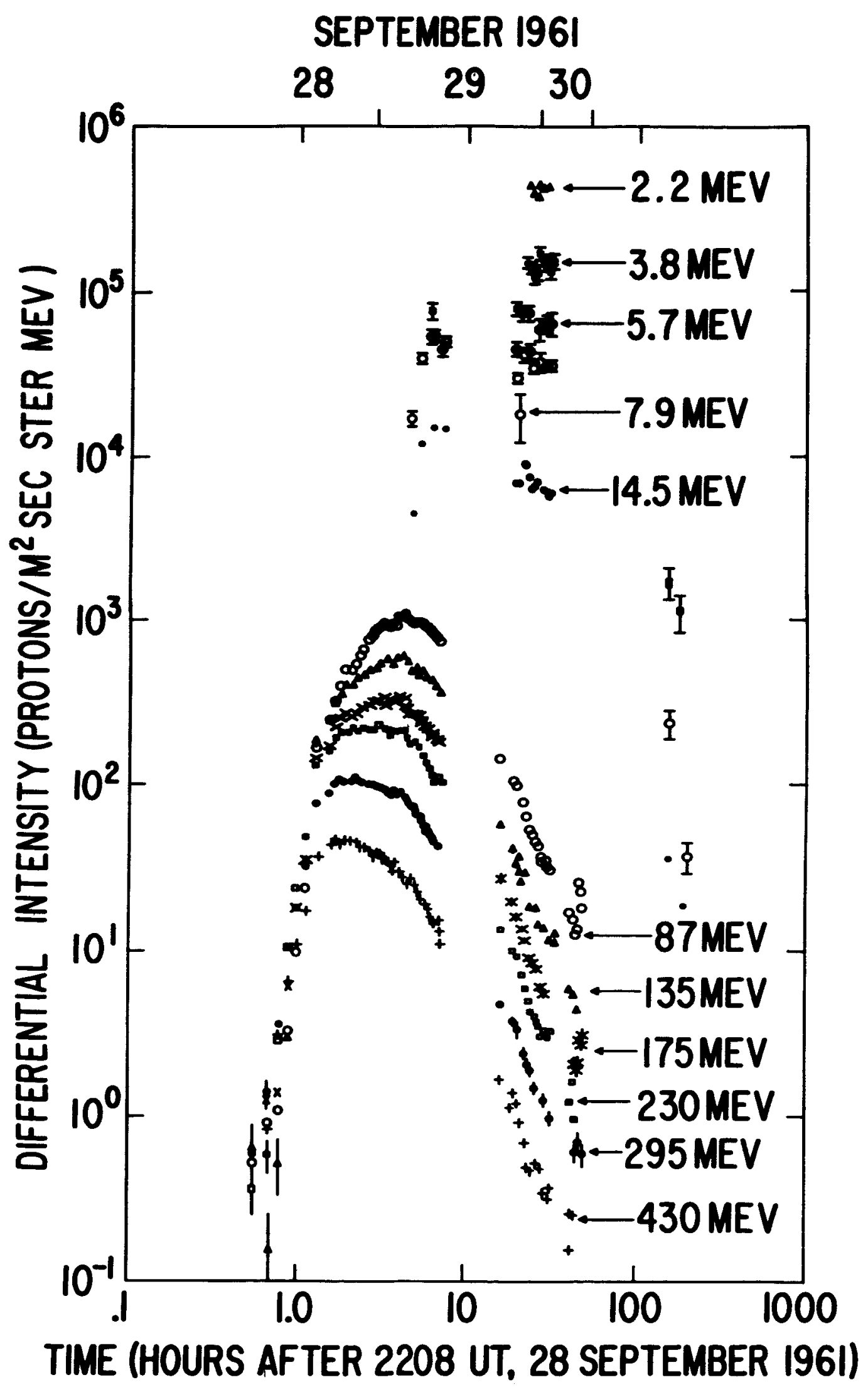

FIG. 3 


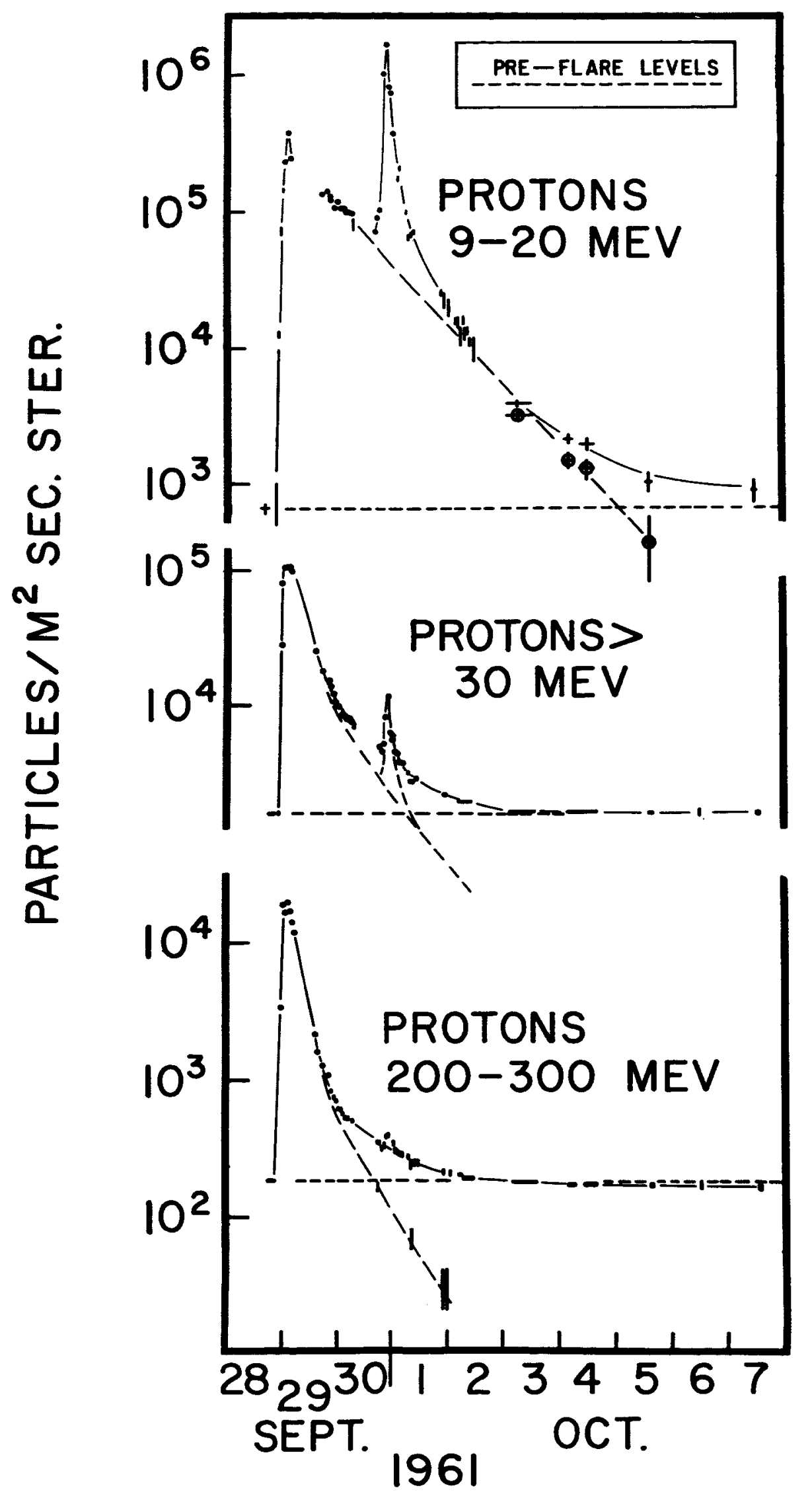

FIG . 4 


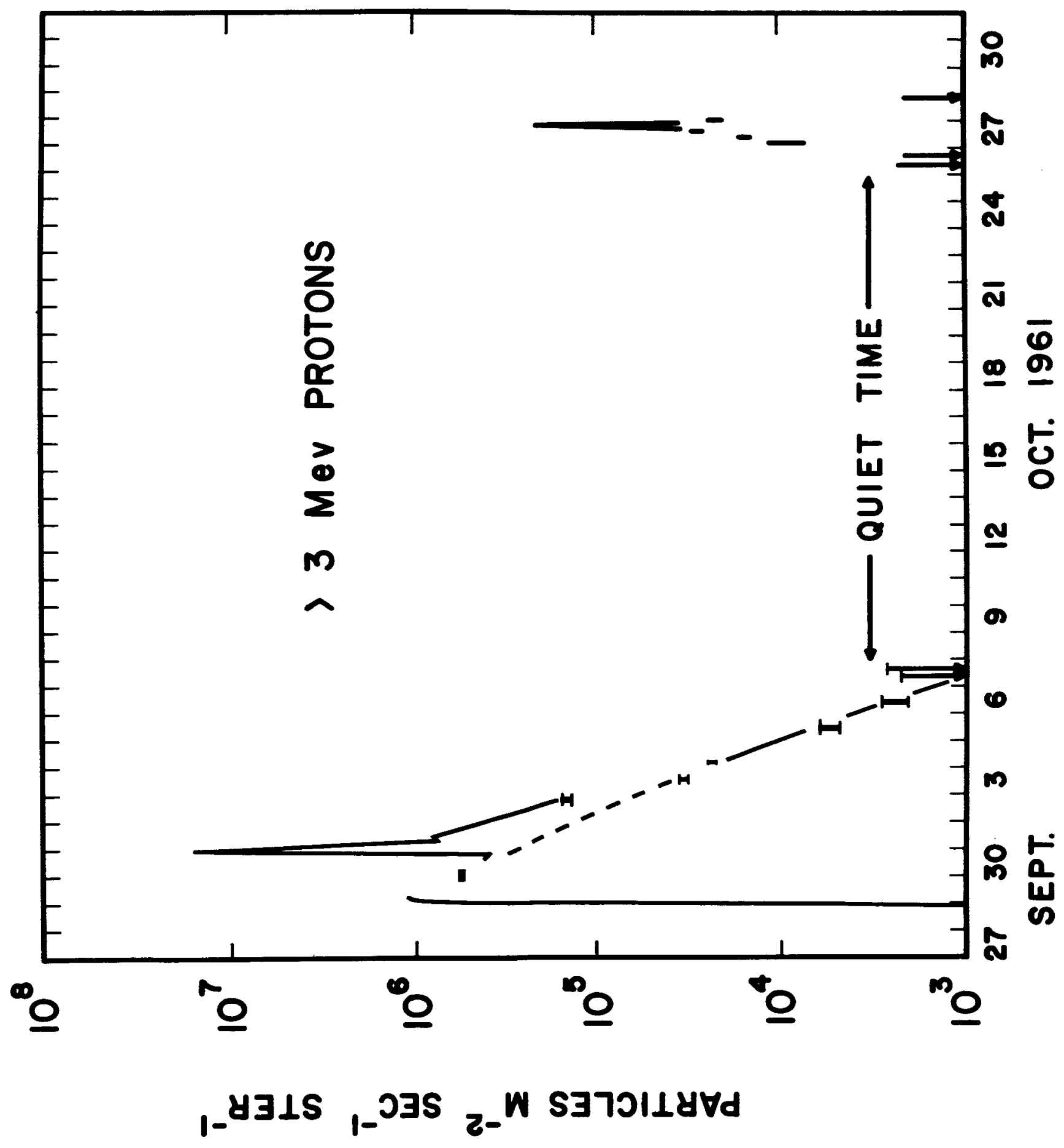

FIG. 5 


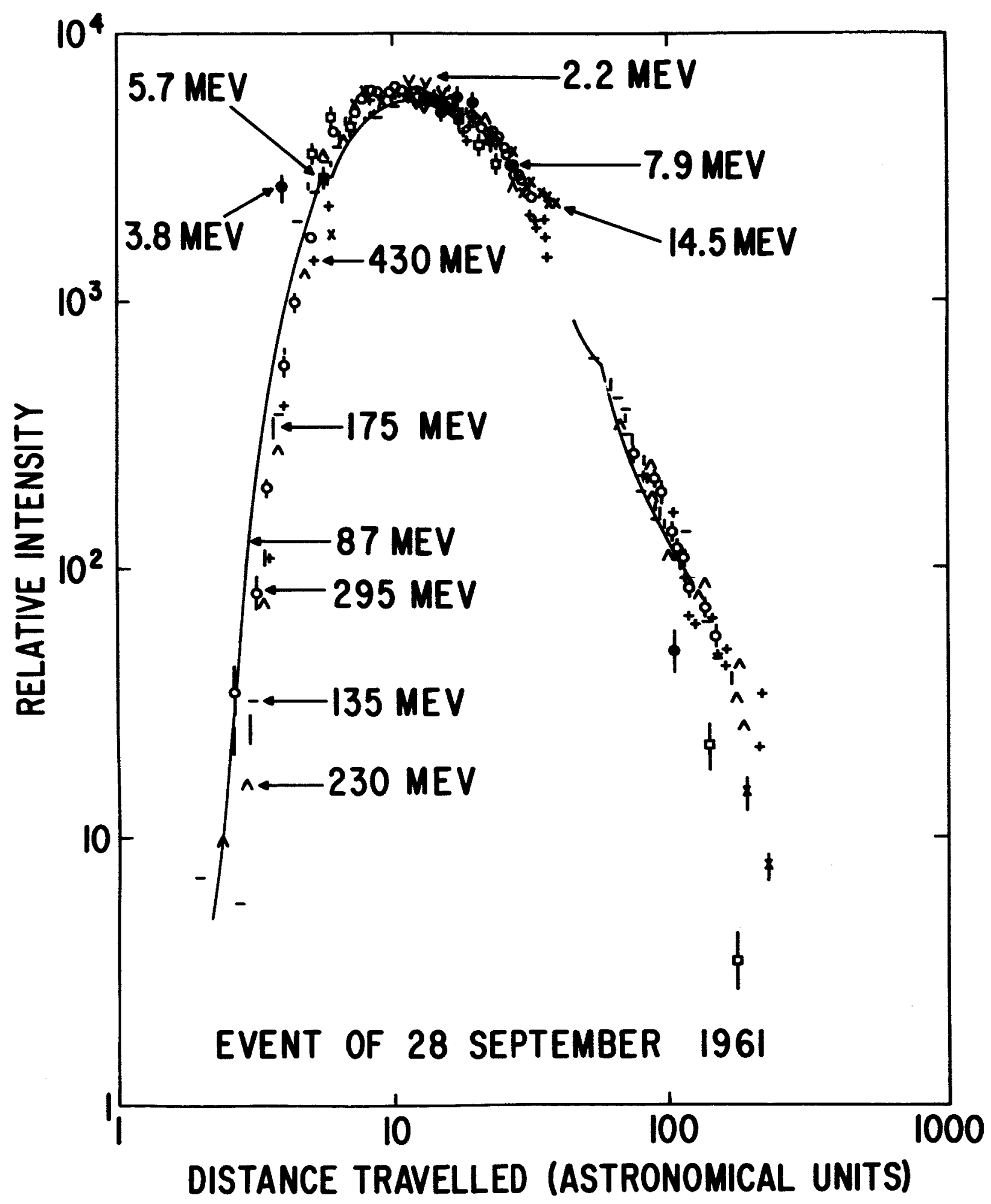

FIG. 6 


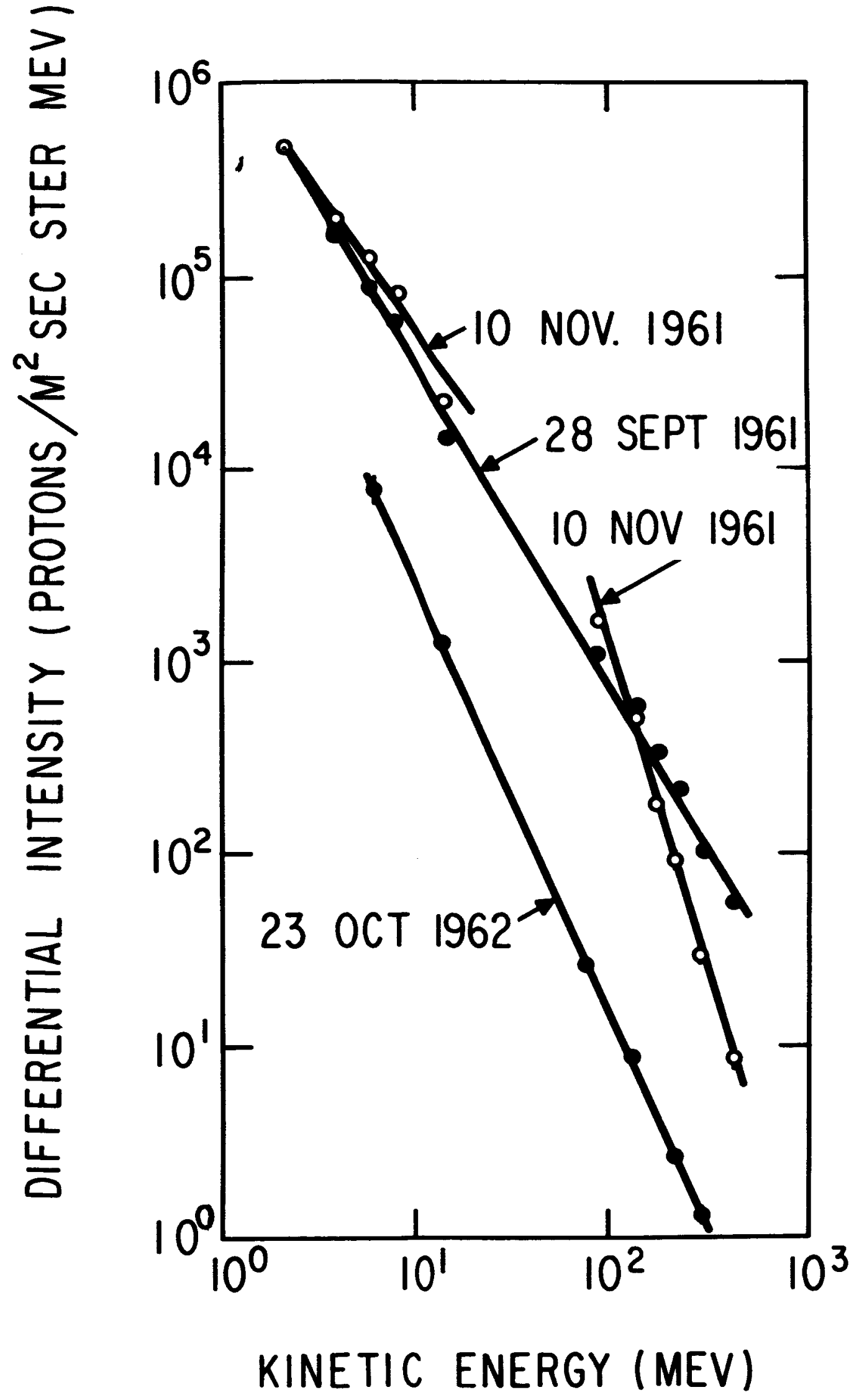

FIG. 7 


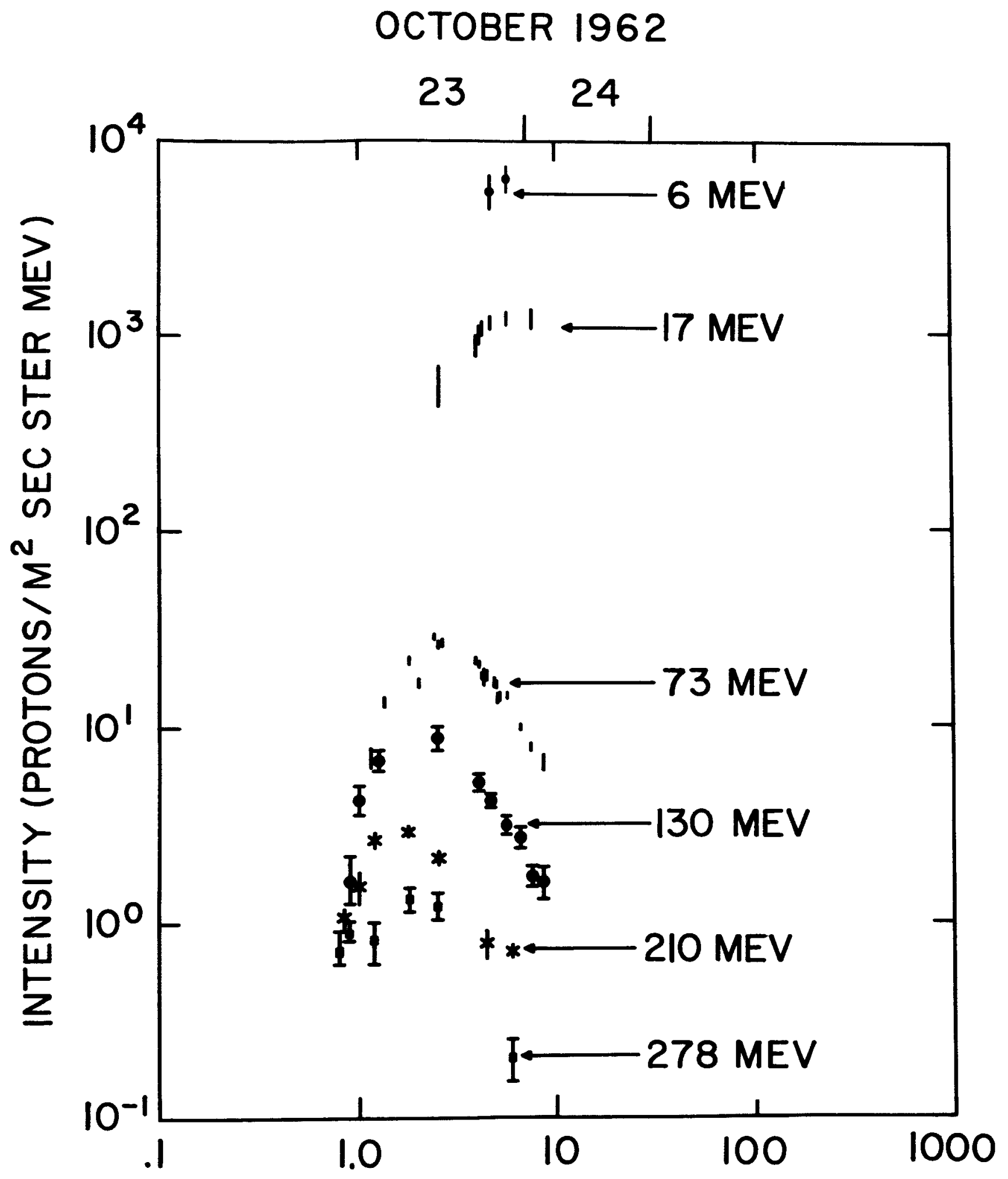

TIME (HOURS AFTER I648UT, 23 OCTOBER 1962) 


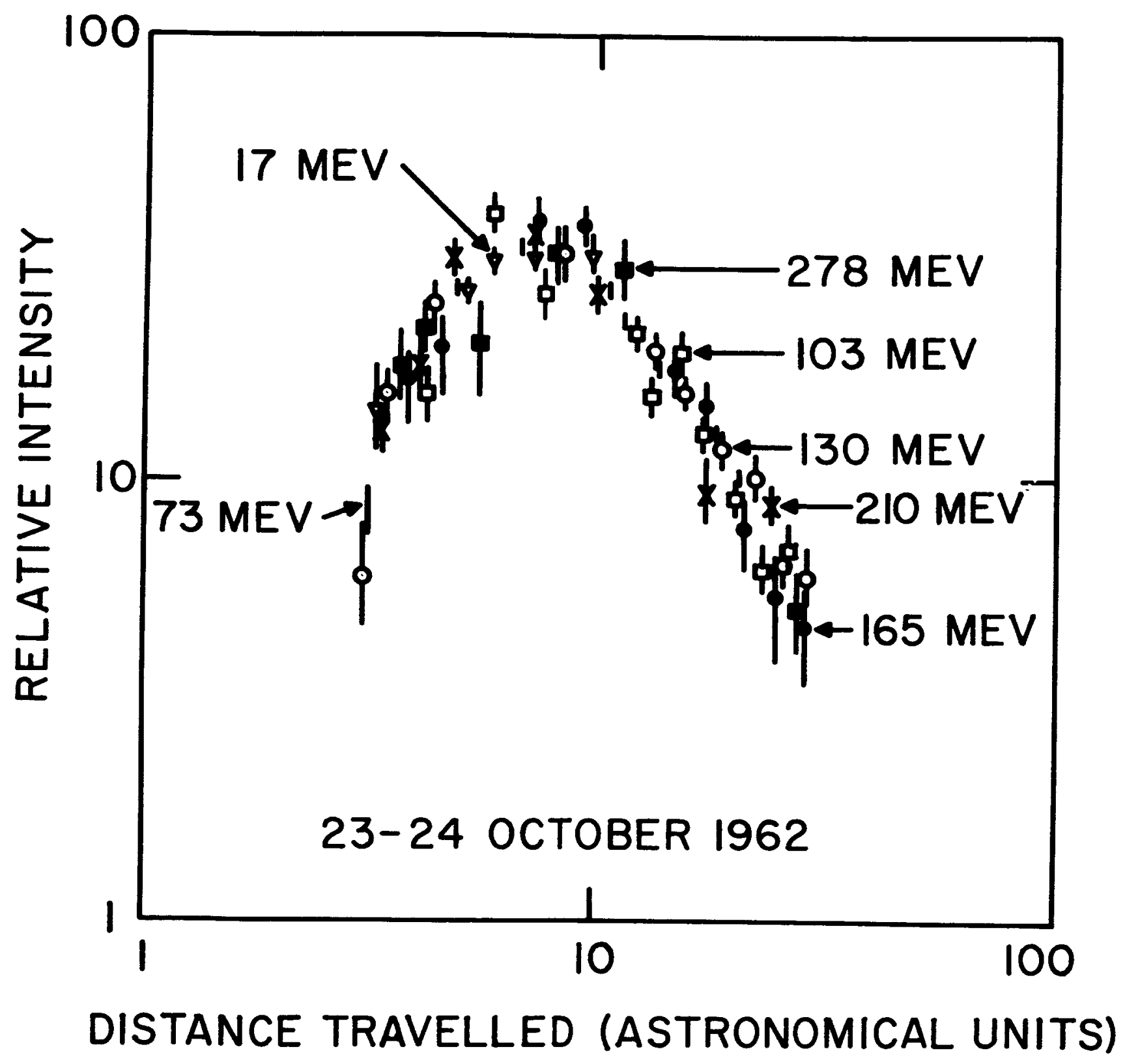

FIG. 9 


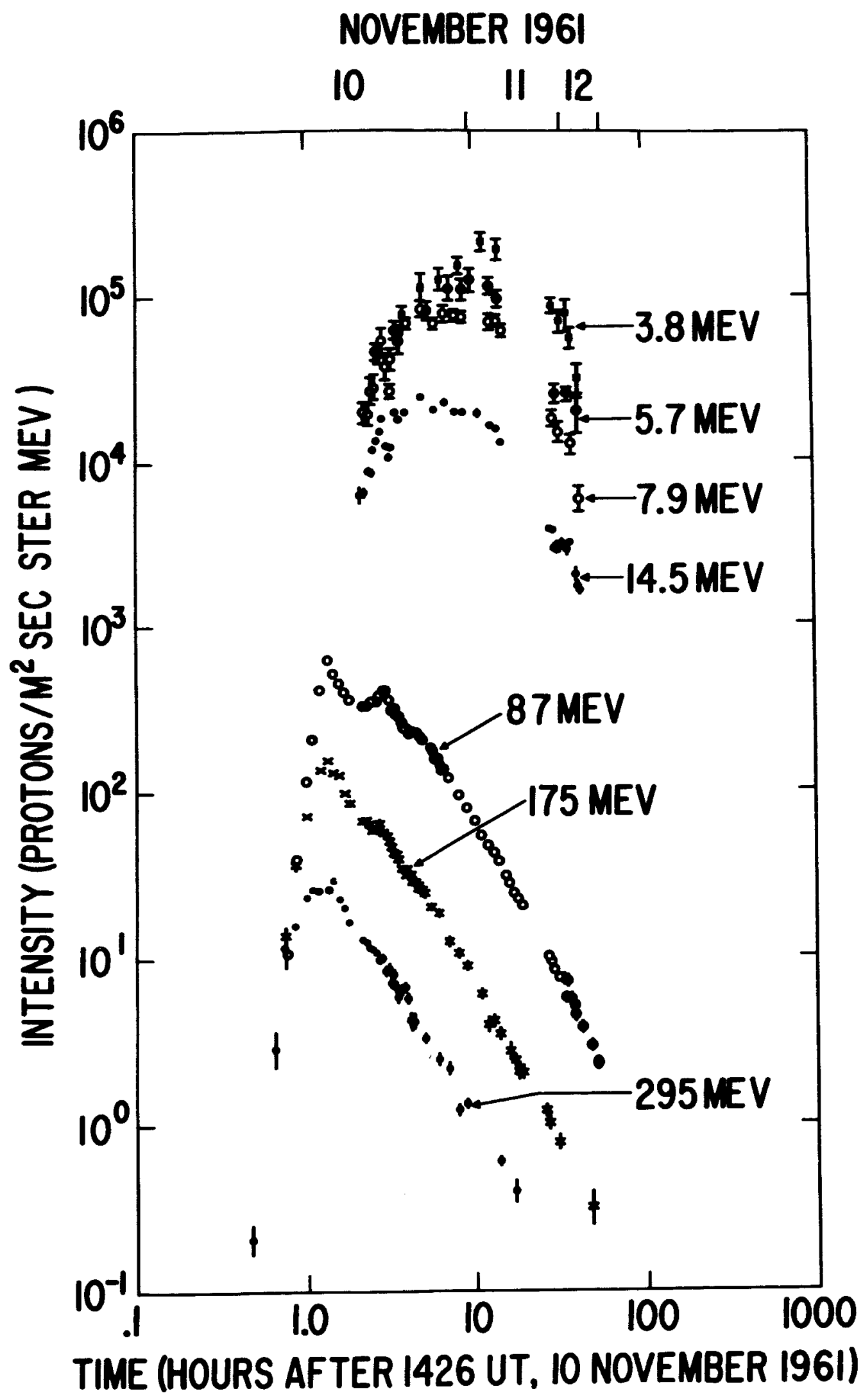

FIG. 10 


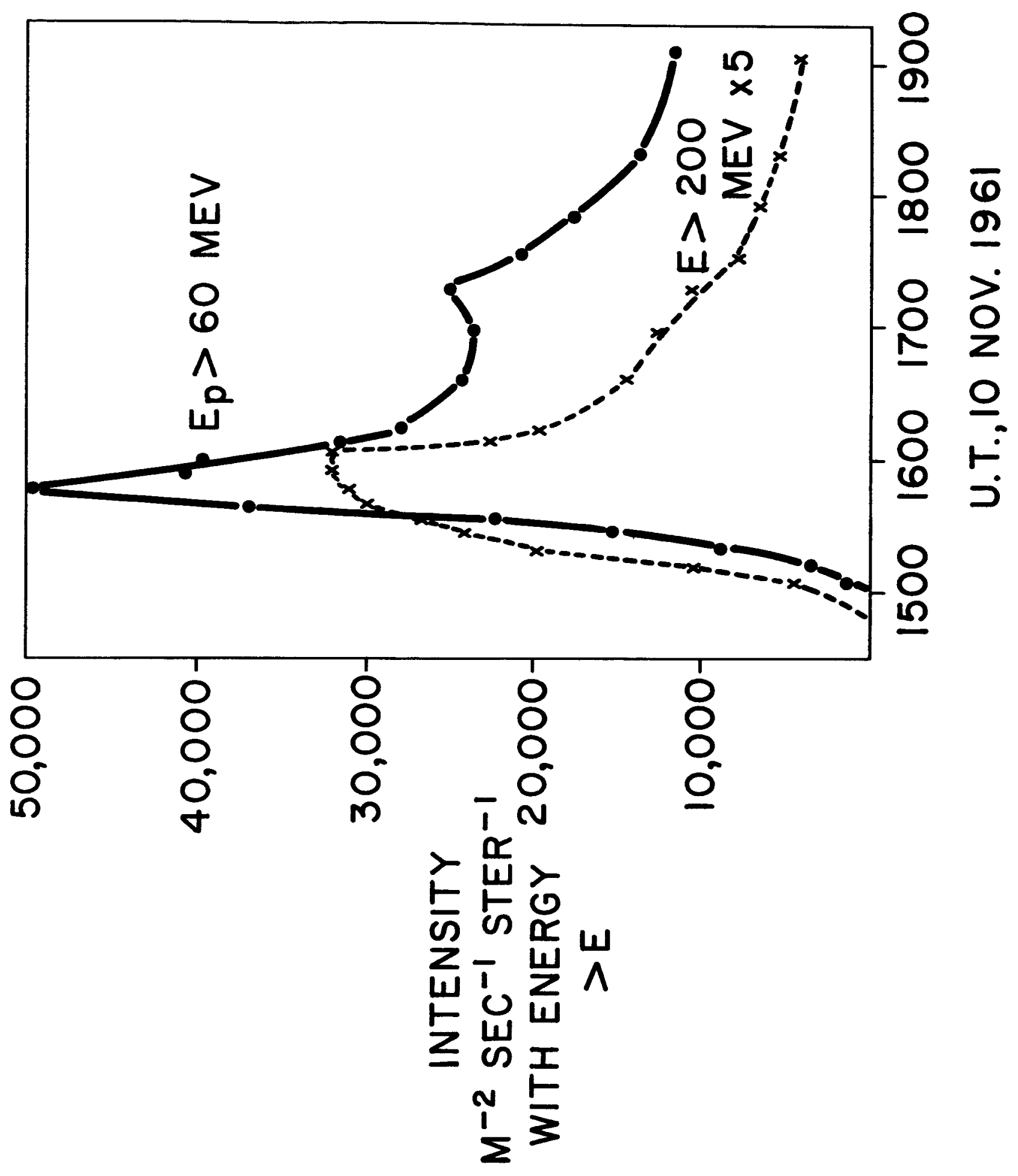

FIG. 11 


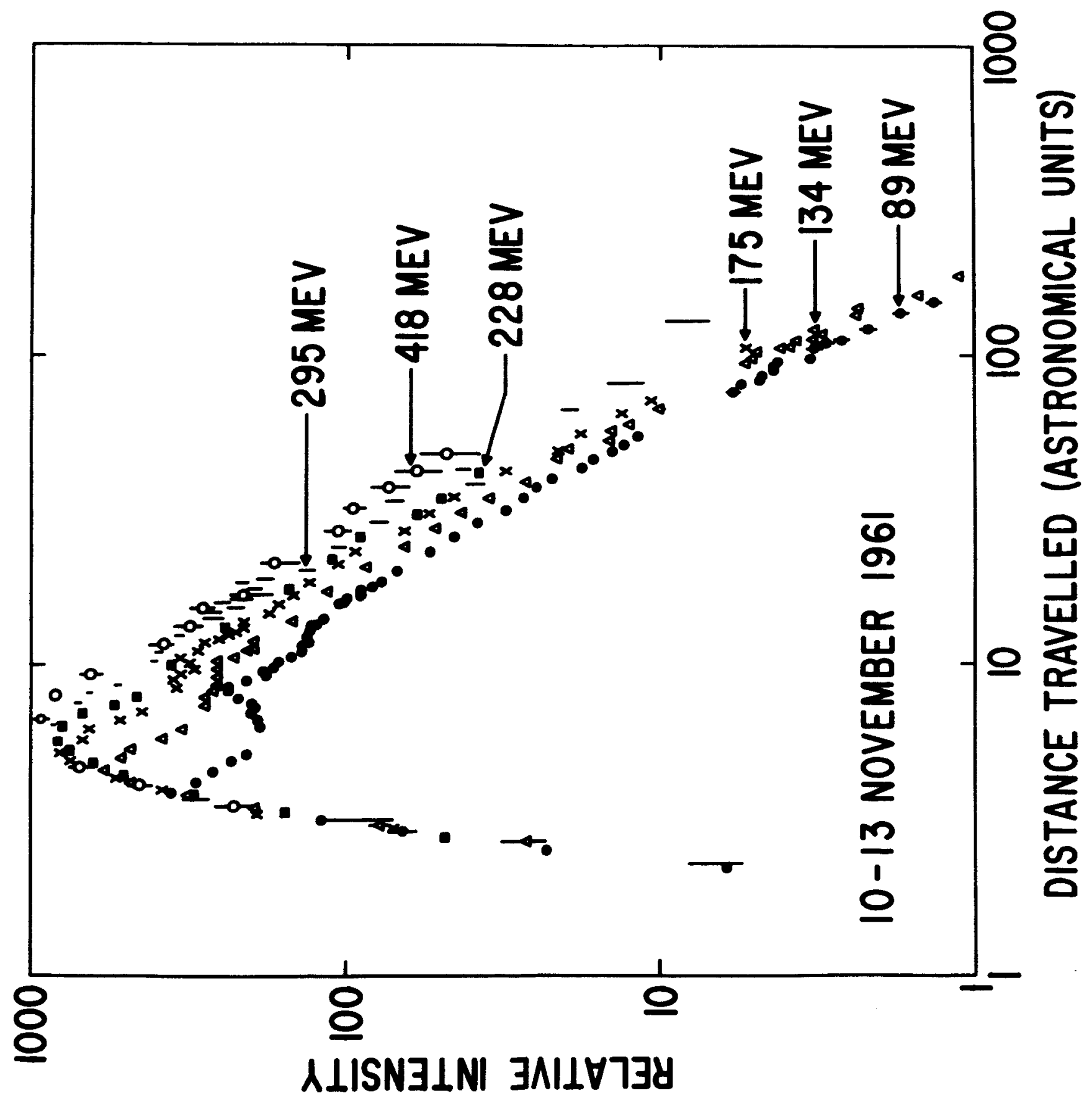

FIG. $12 \mathrm{a}$ 


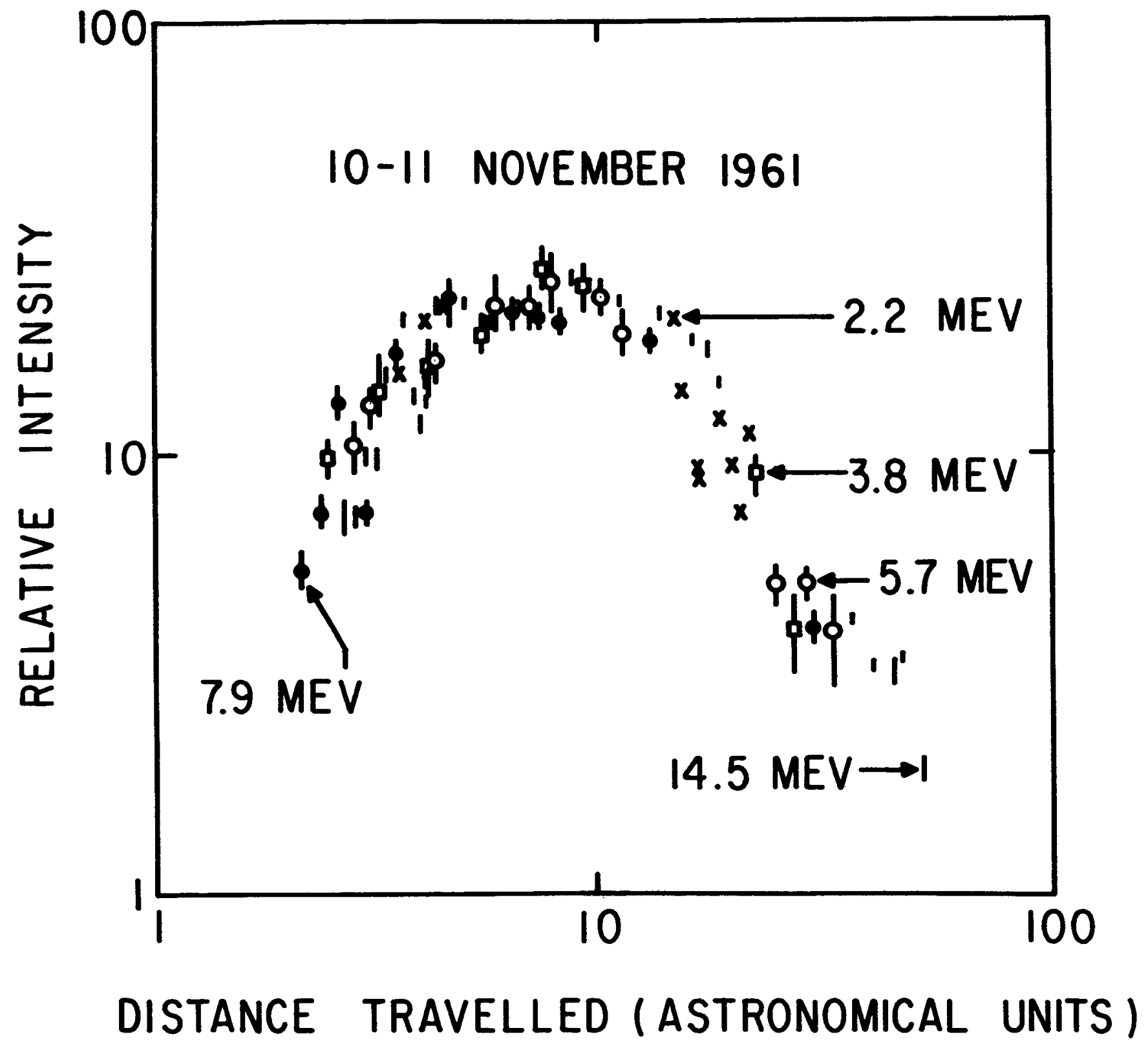

FIG. 12b 


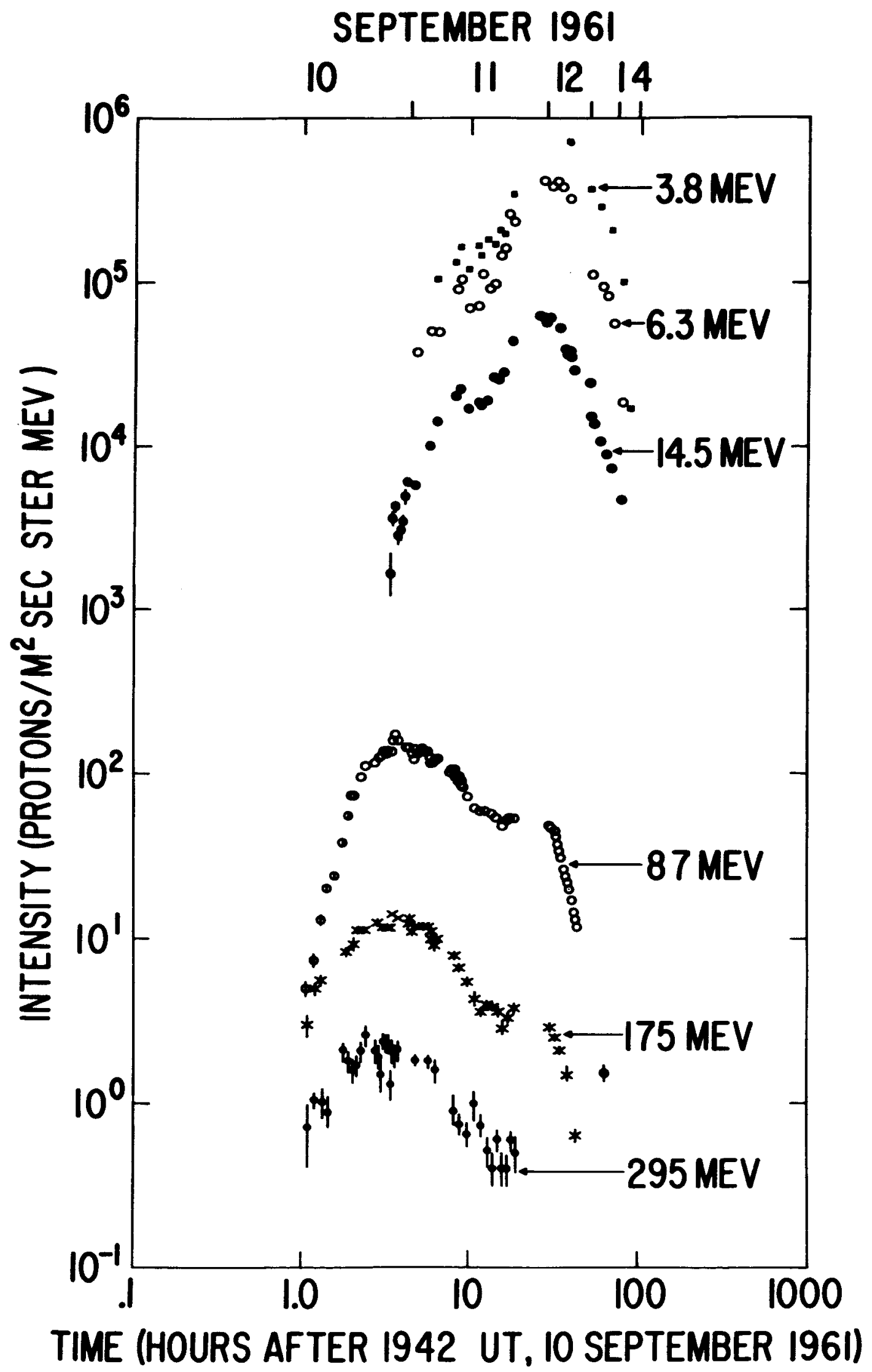

FIG. 13 


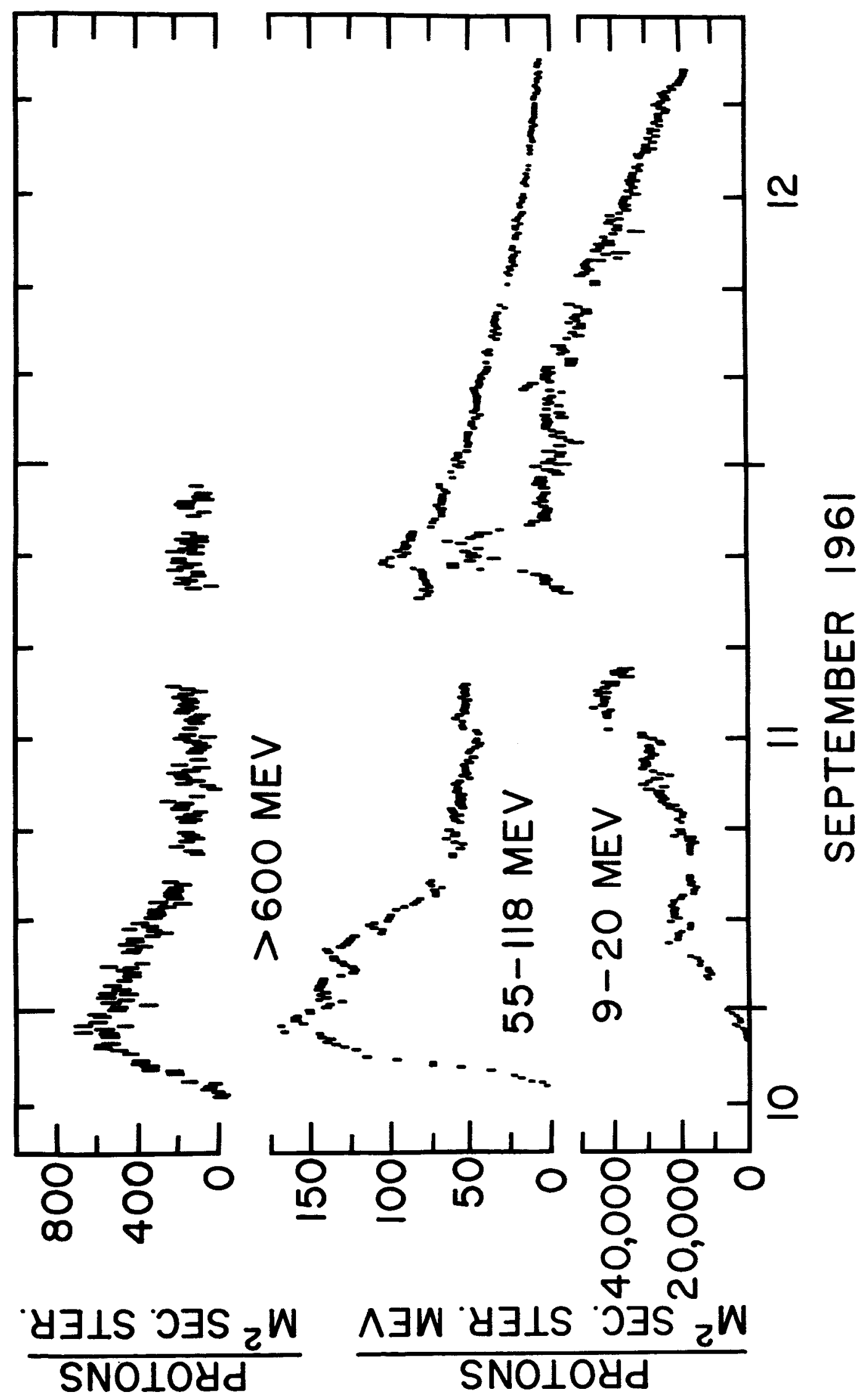

FIG. 14 


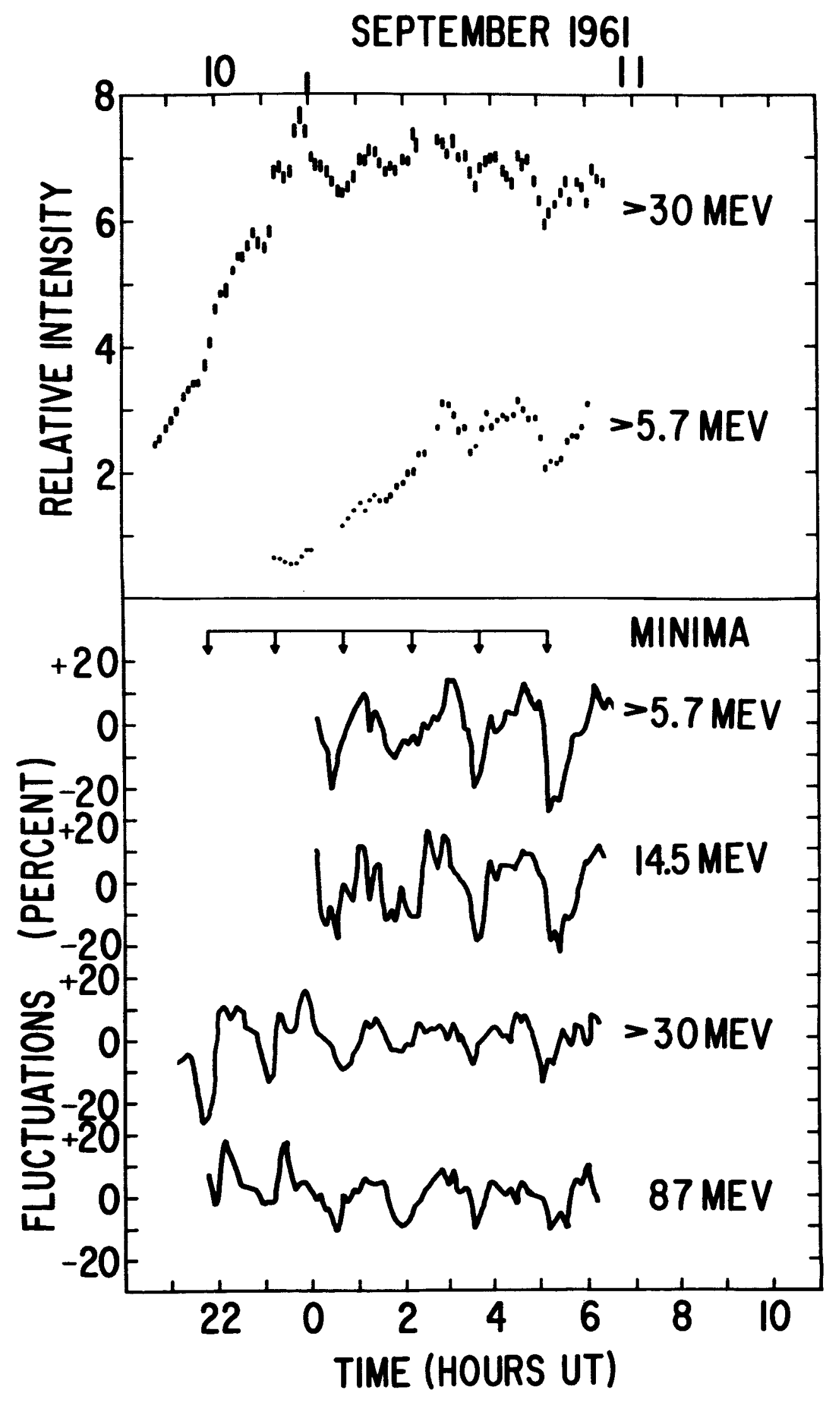

FIG. 15 


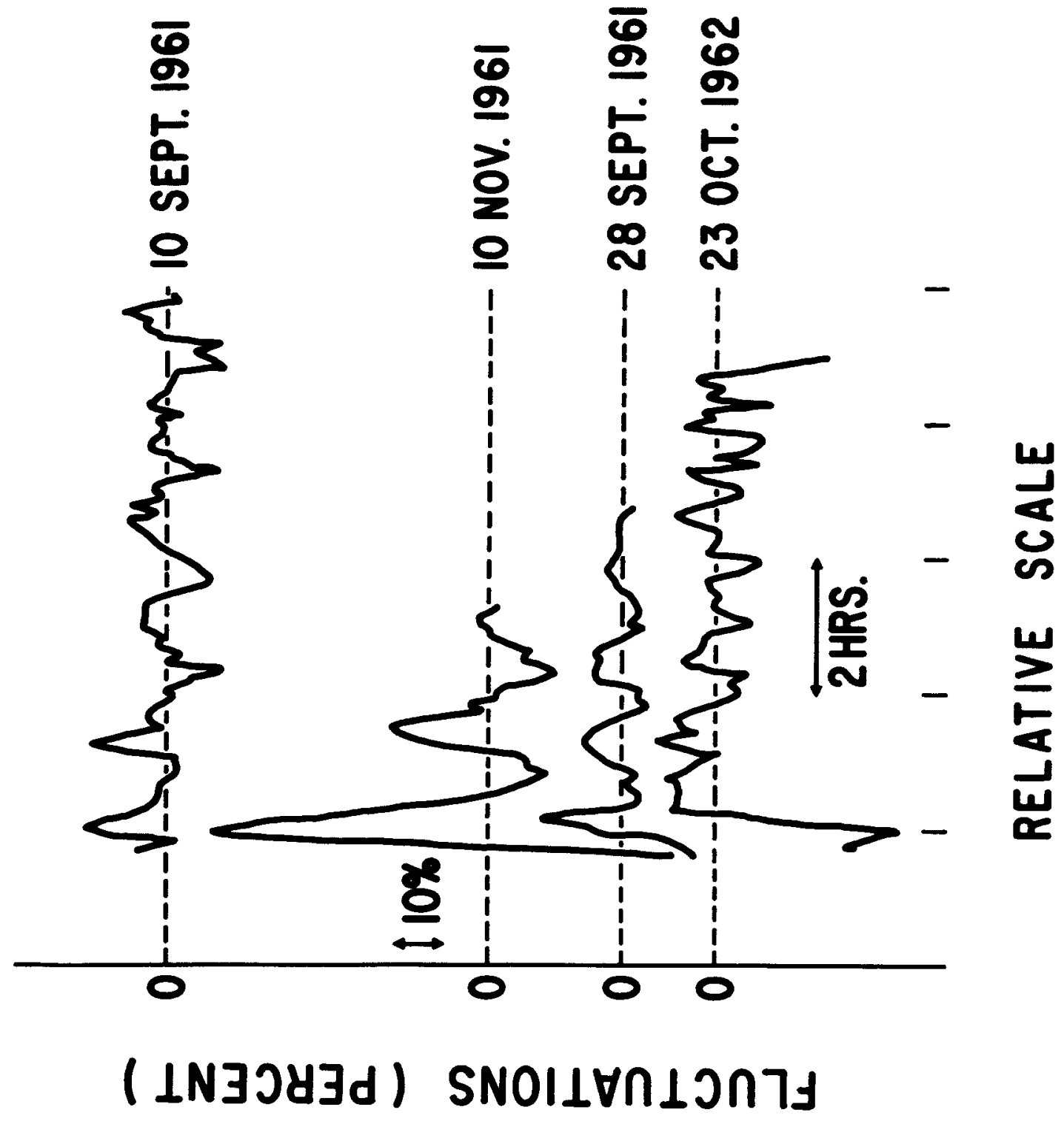

FIG. 16 

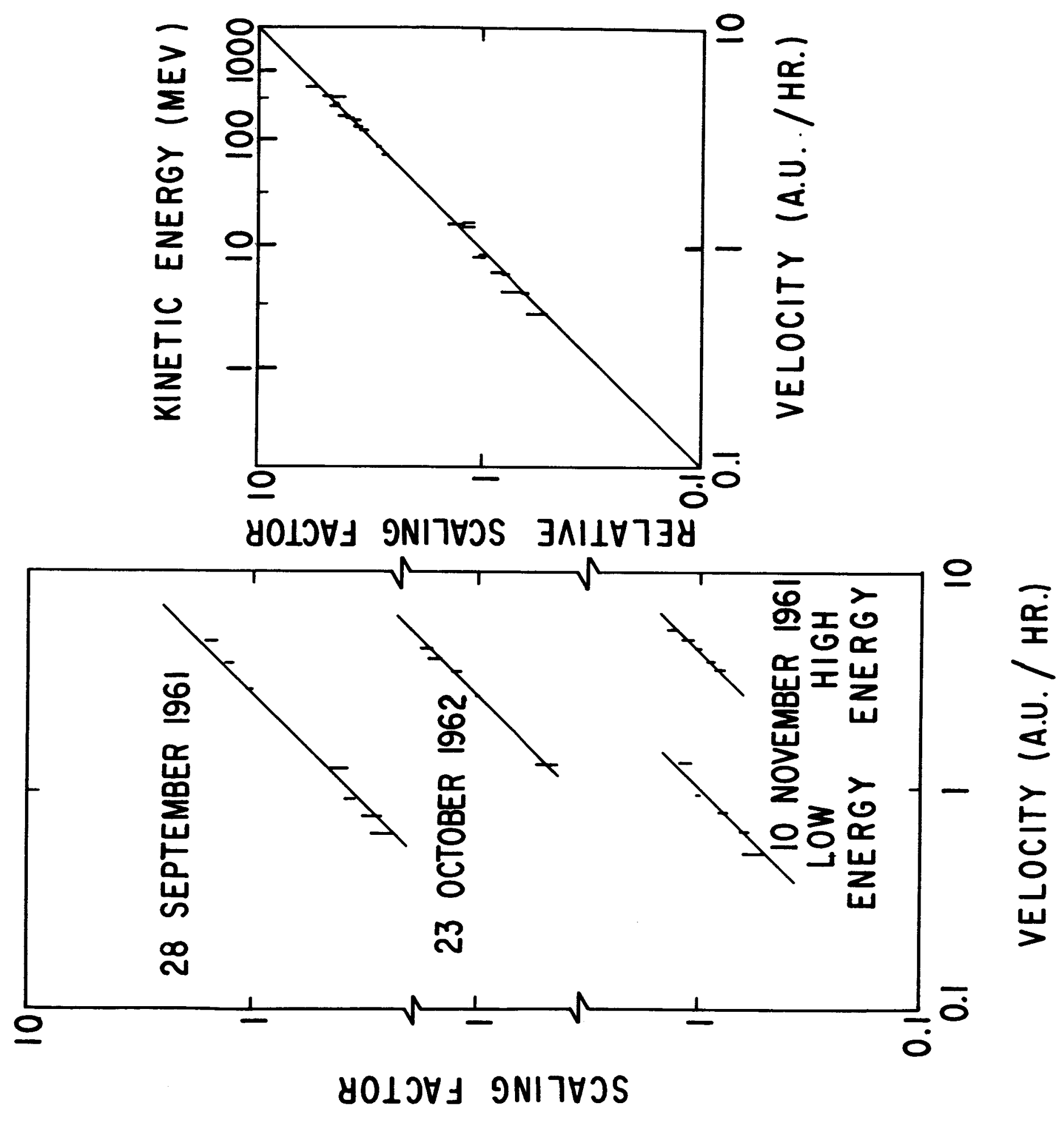

FIG. $17 \mathrm{a} \& 17 \mathrm{~b}$ 


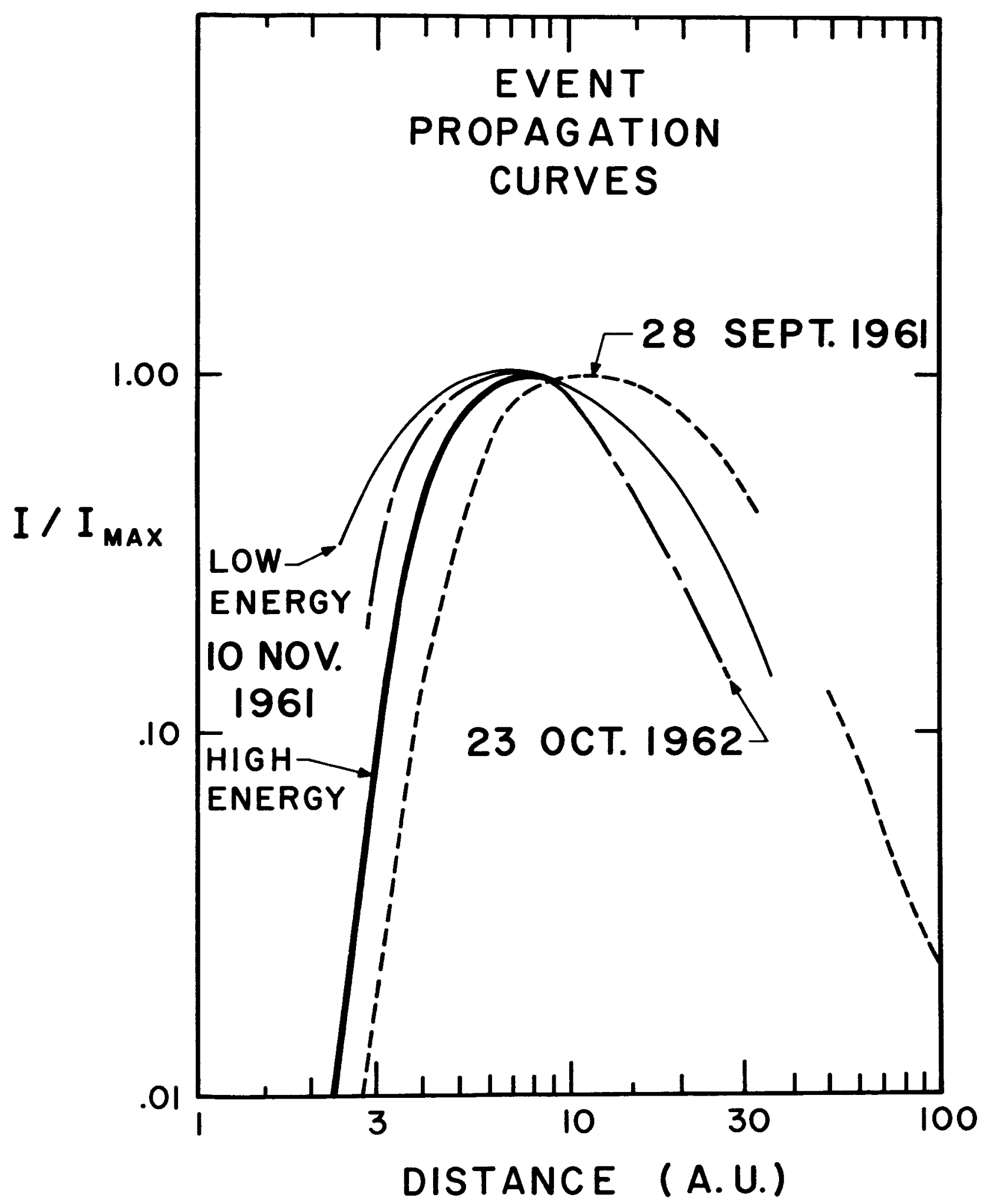

FIG. 18 